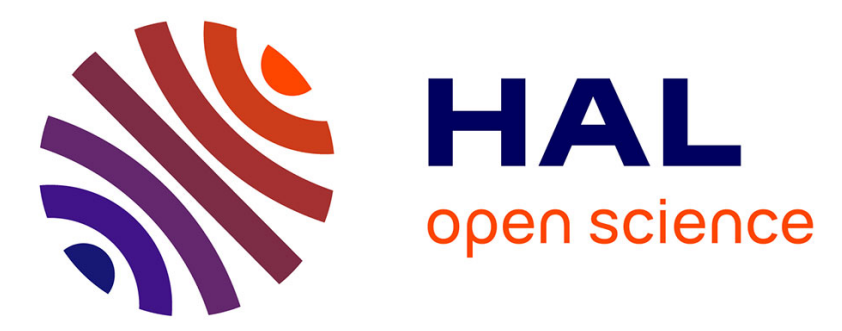

\title{
A Graphical Model Based on Performance Shaping Factors for Assessing Human Reliability
}

Subeer Rangra, Mohamed Sallak, Walter Schön, Frédéric Vanderhaegen

\section{To cite this version:}

Subeer Rangra, Mohamed Sallak, Walter Schön, Frédéric Vanderhaegen. A Graphical Model Based on Performance Shaping Factors for Assessing Human Reliability. IEEE Transactions on Reliability, 2017, 66 (4), pp.1120-1143. 10.1109/TR.2017.2755543 . hal-01668143

\section{HAL Id: hal-01668143 \\ https://hal.science/hal-01668143}

Submitted on 19 Dec 2017

HAL is a multi-disciplinary open access archive for the deposit and dissemination of scientific research documents, whether they are published or not. The documents may come from teaching and research institutions in France or abroad, or from public or private research centers.
L'archive ouverte pluridisciplinaire HAL, est destinée au dépôt et à la diffusion de documents scientifiques de niveau recherche, publiés ou non, émanant des établissements d'enseignement et de recherche français ou étrangers, des laboratoires publics ou privés. 


\title{
A graphical model based on performance shaping factors for assessing human reliability
}

\author{
Subeer Rangra, Mohamed Sallak, Walter Schön and Frédéric Vanderhaegen
}

\begin{abstract}
Human reliability assessment (HRA) is an aspect of risk analysis concerned with identifying, analyzing and quantifying the causes, contributions and occurrence of human failures. Applications of existing HRA methods are often domain-specific, and difficult to implement even for experts. Also, due to the lack of empirical data, managing uncertainty is important, if not essential. In view of such limitations, we propose a new and comprehensive HRA methodology acronymed 'PRELUDE' (Performance shaping factor based human REliability assessment using vaLUation-baseD systEms). It is a quantitative and qualitative HRA methodology, applied to railway operations. The qualitative part characterizes a safety critical situation using Performance Shaping Factors (PSFs). The PSFs are identified from domain specific human factors and PSF-based studies. The quantitative proposition is a framework of a graphical model (Valuation-based System) and belief functions theory. Appropriate representation and handling of all types of uncertainties, and combination of conflicting expert opinions is considered in this framework. To aid in the choice of appropriate combination method, combined expert data is discussed and compared using quantitative metrics. PRELUDE allows quantifying a human failure event given an operational context. Sensitivity analysis is used to establish a priority ranking among the PSFs. Finally, application on a railway accident scenario describes usage and applicability of our proposition.
\end{abstract}

\section{Index Terms}

human reliability analysis, performance shaping factors, human factors, railway accident, valuation-based Systems, belief functions theory

\section{ACRONYMS}

ATHEANA A Technique for Human Error Analysis

AWS Automatic Warning Systems

BBN Bayesian Belief Networks

BFT Belief Functions Theory

BPA Basic Probability Assignments

CPT Conditional Probability Tables

CREAM Cognitive Reliability and Error Analysis Method

EC Error Contexts

EN Evidential Network

EUAR European Union Agency for Railways

S. Rangra, M. Sallak and W. Schön are with Computer Engineering Department at Sorbonne Universités, Université de Technologie de Compiègne, CNRS, Heudiasyc UMR 7253, CS 60 319, 60203 Compiègne cedex, France, (email: subeer.rangra@utc.fr, mohamed.sallak@utc.fr)

F. Vanderhaegen is with Department of Automation and Control at Université de Valenciennes et du Hainaut-Cambrésis, CNRS, LAMIH UMR 8201, F-59313, Valenciennes, France 
FTA Fault Tree Analysis

GTT Generic Task Type

HEP Human Error Probability

HEART Human Error Assessment and Reduction Technique

HERA Human Event Repository and Analysis

HFE Human Failure Event

HFTC Human Failure Type Context

HMI Human Machine Interface

HRA Human Reliability Analysis

HSI Human System Interface

HuPeROI Human Performance Railway Operational Index

MERMOS Méthode d'Evaluation de la Réalisation des Missions Opérateurs pour la Sûreté (French for Assessment Method for the Performance of Safety Operation)

NARA Nuclear Action Reliability Assessment

PRA Probabilistic Risk Assessment

PRELUDE Performance shaping factor based human reliability assessment using valuation-based systems

PIF Performance Influencing Factors

PRELUDE Performance shaping factor based human REliability assessment using vaLUation-baseD systEms

PSF Performance Shaping Factor

RARA Rail Action Reliability Assessment

SPAR-H Standardized Plant Analysis Risk Model - Human reliability analysis

THERP Technique for Human Error Rate Prediction

TRACEr The technique for the retrospective and predictive analysis of cognitive errors

VBS Valuation-Based Systems

VN Valuation Networks

\section{INTRODUCTION}

W UMAn reliability Analysis (HRA) methods have been proposed to systematically incorporate human as part of a PRA (Probabilistic Risk Assessment) activity [1] [2]. Over the years, research and industrial usage has driven various changes in HRA methods and underlying techniques. These changes are often categorized into generations, the 'first' - HEART, THERP, SPAR-H, etc. and the 'second' - ATHEANA, MERMOS, CREAM, etc. The first generation methods have been developed with features broadly to THERP such as task analysis, nominal probabilities for human failures, and adjustment to consider different conditions, etc. The second generation aimed for a lesser focus on individual errors, and higher on determining the factors and conditions around said errors, i.e. 'intrinsically unsafe situation', and other emphasized cognitive model-based methods aiming for a complete capture of human performance [3]. Nevertheless, this classification is often not sufficient and a clear identification of desired and valid techniques is not straightforward [4] [5].

There are varied interpretations and propositions to the problem of human error [6]. For example, contrary to technical component reliability, human reliability is not static but evolves dynamically [7]. A prediction of human reliability should in that case include models which predict human actions by modeling the act of merging knowledge gained by a human in a situation [8]. The management thereof focuses on adjustable human-machine cooperation [9], supporting a human in reasoning/decision-making, and system resilience [10]. However, generally in the context of HRA, human reliability is often analyzed and defined in terms of the causes of human behavioral or functional dysfunction [4]. Theses causes are often characterized as Performance Shaping Factor (PSF). In most HRA methods, PSFs (or a variant thereof) are used to identify contributors to human errors and to provide a basis for quantifying those contributors systematically [11]. More specifically, PSFs allow the consideration of human's own characteristics along with the context and environment which affect the performance in a negative or 
positive manner [12]. First generation HRA methods employed PSFs as 'modification factors' to expected human performance (for HRA - nominal human error probabilities). Most second generation methods also agree that PSFs are an integral part of the modeling and characterization of errors and play an important role in the process of human reliability assessment. In general, various recent HRA models, and similar works (viz. Good Practices, SPAR-H, CREAM, 9-Factor Model, etc.) analyzed in [13] reach a similar conclusion in terms of the need of PSFs for HRA. The PSFs can then also be viewed as contributing factors that affect an operator's performance, which in turn leads to an accident/incident. In [14], it is concluded that 18 PSFs were responsible for more than $80 \%$ of the railway accidents they analyzed. A follow-up study [15] with additional data concluded that 12 PSFs, account for more than $90 \%$ of the accidents and incidents. This dual nature of PSFs in characterizing accident safety critical contexts (frequentist) and accounting for human and situational aspects (shaping human performance in general) make them an ideal candidate for risk-based decision making.

In the second generation of methods the focus was diverted from individual errors to cognitive aspects and the determination of factors (PSFs or PSF-like) in combination lead to an intrinsically unsafe situation [16]. Data on identification of contextual information itself can present challenges of uncertainty in the model [17]. Such a situation can be defined by one or more PSFs [18]. In ATHEANA, Error-forcing context (EFC) is defined as "... particular combinations of PSFs and plant conditions creating an environment in which unsafe actions are more likely to occur." EFC aims to present the experts potential interactions among the set of factors that are significantly different than the usual influence of individual factors. More recently in [19], ECs (Error Contexts) are derived from empirical data, they are defined as "certain combinations of PSFs those are more likely to produce human errors than the individual PSFs acting alone." It is further argued in [20] that such a collection of PSFs aids the experts in the elicitation process. This collection of PSFs as particular sets, characterizing a particular context or situation is also stated to be easier for usage [19], [21] [22]. In [23], EFCs are appreciate for providing HEP estimates based on realistic causes and an invaluable input for second generation methods.

For the quantification of rare events such as human failures managing uncertainty in data is an important and challenging task [24] [25] [17]. As remarked in [6]: "lack of data is probably the single most important factor impeding the development of human reliability indices." Rather recently, this issue was also recognized in the SPAR-H guide [26]. Some key aspects of quantifying such rate events in a PRA are: evaluation, propagation, combination and adequate representation [27]. Thus, towards the objectives of an accurate representation and subsequent evaluation, it is frequently classified by its source. The one originating from natural randomness is called aleatory and the other originating from a lack of information is termed epistemic. Aleatory is independent of the analyst's knowledge of the system and is inherent in nature, therefore is sometimes also called irreducible. Epistemic uncertainty on the other hand can be reduced by improving the model of the system under analysis. The interest in this classification is to mainly understand what is reducible and what is not. This classification is often used in the domain of risk analysis [28] [29]. Apart from traditional way of using classical probability theory [30], there are some alternative ways to represent both types of uncertainties, such as Fuzzy sets and theory [17], imprecise (interval) probability, possibility, belief function theory, etc. [25] [31]. Further, as concluded in [28] only the probability bound (latter two) approaches provide an easy interpretation in a practical decision-making context. The author further argues to "make these alternative approaches operational in a practical decision-making context, the probability bounds need to be highlighted, not the underlying theories."

When working with a lack of empirical data, concepts such as expert elicitation, conditional data, prior probabilities and combination thereof can be employed. Probabilistic graphical modeling frameworks are particularly easy to use in interaction with domain experts and are more expressive than traditional approaches [32]. Using expert systems for quantitative HRA can be helpful to improve the transparency and repeatability of assessments [33]. The usage of Bayesian network in the domain of reliability [34], and particularly HRA has seen significant growth in recent years [35] [36]. For HRA applications, this variable of interest is a specific failure event (e.g. an HFE: Human Failure Event) or a generic failure event, or system level inference such as an accident. The intermediate nodes or parent nodes (parent and child for multi-level BBN) are generally PSFs [36]. The objective is to compute probability of the occurrence of HFE (e.g. HFE in state true). To do that, once the variables and their states (or values) are identified, formal conditional relations need to be defined. These relations aim to define the 
PSFs influencing human performance towards a certain task/function or failure thereof (HFE). Towards this task, data-informed approaches to 'build' PSF-HFE relations have also seen recent popularity. In BBNs with discrete state nodes, these relations are represented as Conditional Probability Distributions of each child node's state for each possible combination of the parents' states and defined using CPTs (Conditional Probability Tables) [36]. The CPTs formally define these relations, and can vary within the interval $[0,1]$. The exact values thereof can define a strong or a weak influence among variables. This data can come from empirical sources as presented in [19]. In domains where empirical databases of human failures are rare or are not suitable for formally building such relations, expert opinion remains a common and acceptable source [37] [38] [36], and rail transport is not an exception. Such frameworks, thus, not only provide an expressive reasoning framework, but also allow for the possibility to employ expert and empirical data towards HRA objectives.

For a domain like railway, experts can be elicited for data on conditional elements, i.e. failure(s) and factor(s) (HFEs and PSFs). In [38] this relational data represents the interpretation of multiple experts' estimates as specific probability values on the set of specific states of HFEs and PSFs. In ATHEANA's expert elicitation process [20] the HFEs are 'unsafe actions' (UAs), which can be a collection of HFEs. The information, thus desired from an expert, is subjective probability on conditional elements of human performance. Generally, in such cases multiple experts are consulted, methods to combine expert judgments are thus needed. Some use a group consensus approach (a behavioral approach) towards quantifying human actions and treating uncertainty [20]. However, for quantitative applications it is generally agreed that mathematical approaches for aggregating expert opinions yield more accurate results than behavioral approaches [39]. In [38], such a formal aggregation (hereafter referred as combination) of expert estimated probabilities using a Bayesian approach is presented. This combination accounts for difference in an expert's belief (what present work hereafter refers to as conflict) and inherent variability in HEP estimates. However, in such data combination approaches, it is often required to ensure expert estimates are modeled as independent. As concluded in [36], when employing BBN-based or similar approaches, combination of data taken from experts needs more work.

Despite the high share of human errors in rail accidents [40] [41] [42] domain specific methods of risk assessment, considering human factors, towards an operational railway context are very few and rather limited. In some cases, error probability of every human action is assigned a fixed value of $10^{-3}$ [43] [44]. It is said to be a hypothetical value representing a generic estimation of HEP, and the assignment is referred to as "... a non-written agreement." in industrial railway applications [45]. Furthermore, with increasing complexity of systems, such as rail transportation, notions of system-of-systems call for systematic methods to address human factors and safety issues [46]. Nevertheless, some relevant works in the railway domain are discussed here. A context related HRA model for rail systems is proposed in [44]. They also identified and discussed PSFs and their importance to characterize a context. In [47] a qualitative analysis approach has been proposed to account for certification requirements. Here, the authors argue for the applicability of a PSF-based model in safety analysis of human-barrier interaction. The work in [48] presents a rail industry specific HRA method called Rail Action Reliability Assessment (RARA). It is developed adapting the concepts from the HEART approach [49], such as GTTs, Error Producing Condition, etc. Recently in [50], a PRA of a high-speed railway line, using a MarkovianBayesian network model was proposed. They considered a driver's state as one of the six possible values, three of which were accident states (minor, moderate and severe), the other three distracted, attentive and alert represented states of driver's attention. This, from perspective of PSFs, are considered as levels (or values) of a single PSF (i.e. attention). To support the quantification of human performance for risk assessment objectives, Human Performance Railway Operational Index (HuPeROI), a HRA model, which accounts for PSFs' influence is proposed in [51]. In this approach, expert opinion is needed for PSF measurement, weighing factors and relations for quantification. Further, in [52] after an extensive discussion of specific HRA methods, the author concludes that very few of the analyzed methods were established on the concept of PSFs, while only one (RARA - Rail Action Reliability Assessment) can be argued to be a complete methodology. Moreover, there are very few works dedicated to quantitative assessment of HRA. Irrespective of the approach employed HRA can help in managing and containing the increasing share of human error in transportation accidents. A recent survey by the EUAR's (European Union Agency for Railways; old ERA - European Railway Agency) Human Factors Network [53] and amendments to 
CSM regulation (Common Safety Methods) [54] concur, where, firstly, human factors are recognized to be "main contributors to the systematic failures and systematic faults" and owing to the absence of HRA methods used, call for "a need of information exchange on criteria for risks related to human errors and their assessment."

Table 1 shows a comparison of a few well-known HRA methodologies. Most HRA methods have commonalities such as qualitative and quantitative components. Most of the methods do not integrate empirical data in the quantitative part. This lack of data is managed by either using data from other models, or by eliciting experts, or both. Furthermore, the data obtained from experts is combined, by either using weights/ratings or reaching a consensus. Thus, conflict or lack of knowledge of experts is considered implicitly in the model, explicitly when eliciting the experts. However, as discussed previously (section 1), most of them do not explicitly consider all types of uncertainty. Some recent works (last column of Table 1) have managed this issue by: (i). Using graphical models (BBNs) and (ii). Alternative uncertainty theories such as fuzzy theory. Nevertheless, validation, even for industrial scale models remains a challenge and the domain specific nature of HRA models limit it further.

Thus, the present work focuses on the needs of railway domain. It is also, inspired by the concurrent HRA practices, and aims to address some issues of the HRA methods as resumed in Table 1 . The original contributions of the present work are as follows:

- Guidelines on identification of PSF (rail-specific) and HFE, from human functions and accident analysis reports (most HRA methods provide this step, but are domain-specific in nature, column 2, Table 1 ).

- A quantitative framework of human reliability using a graphical model, which is easy to use by the experts (most use expert elicitation, however, it is not necessarily conditional, or lacks an explicit consideration of conflict or uncertainty in model, column 3, Table 1).

- In the absence of data for the rail domain, expert data can be used, thus a combination and uncertainty management aspect is needed. Here, a formal combination and transformation approach is proposed, to deal with conditional expert data which can work with particularly conflicting opinions and uncertainty (this work makes a distinction between aleatory and epistemic nature of the uncertainties explicitly in the model and results, contrary to most other complete methodologies column 4, Table 11).

- The VBS/BFT framework allowing for imprecision of data in modeling and quantification as imprecise probability intervals (this work makes a distinction between aleatory or epistemic nature of the uncertainties, explicitly in the model and the results, contrary to most other complete methodologies, column 5, Table 11).

- Decision-making by using sensitivity analysis to rank PSFs.

- Applied to a recent high-speed railway accident scenario (most methods are either proposed for the nuclear domain, very few are generic, column 6, Table 1).

Furthermore, all the necessary details are provided in this paper to aid an analyst or an engineer to apply the proposed methodology. It can be applied to other transportation systems such as road vehicles. The rest of this paper is organized as follows: Section 2 starts off with an overview of the PRELUDE methodology. Subsections thereof give the theoretical background of the mathematical framework employed, followed by the main contribution of this work. It is explained and concluded with a discussion on the quantification results. The case study is presented in Section 3, where the proposed methodology is employed for a retrospective analysis of a railway accident scenario. The paper ends with conclusions and perspectives in section 4. 


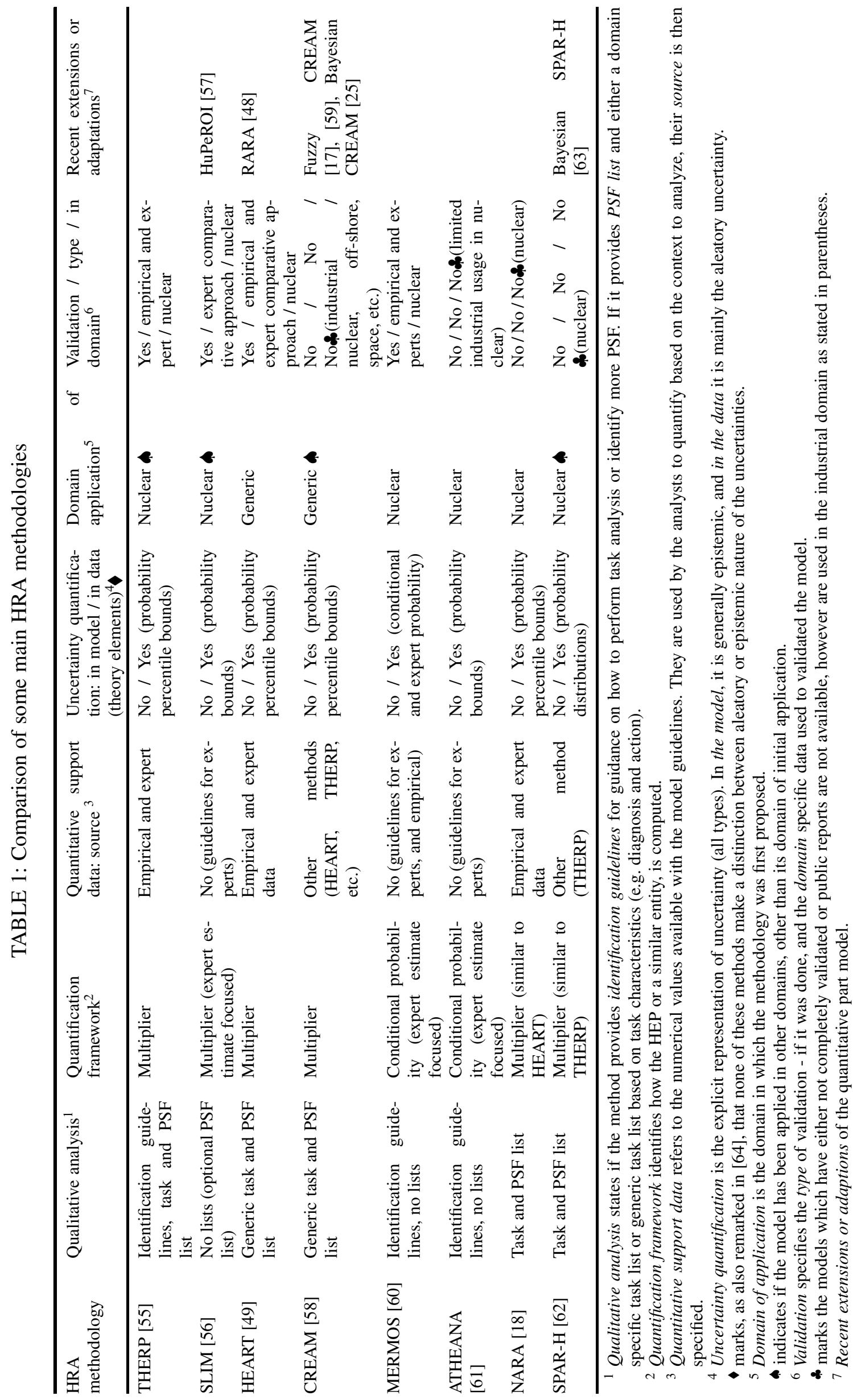




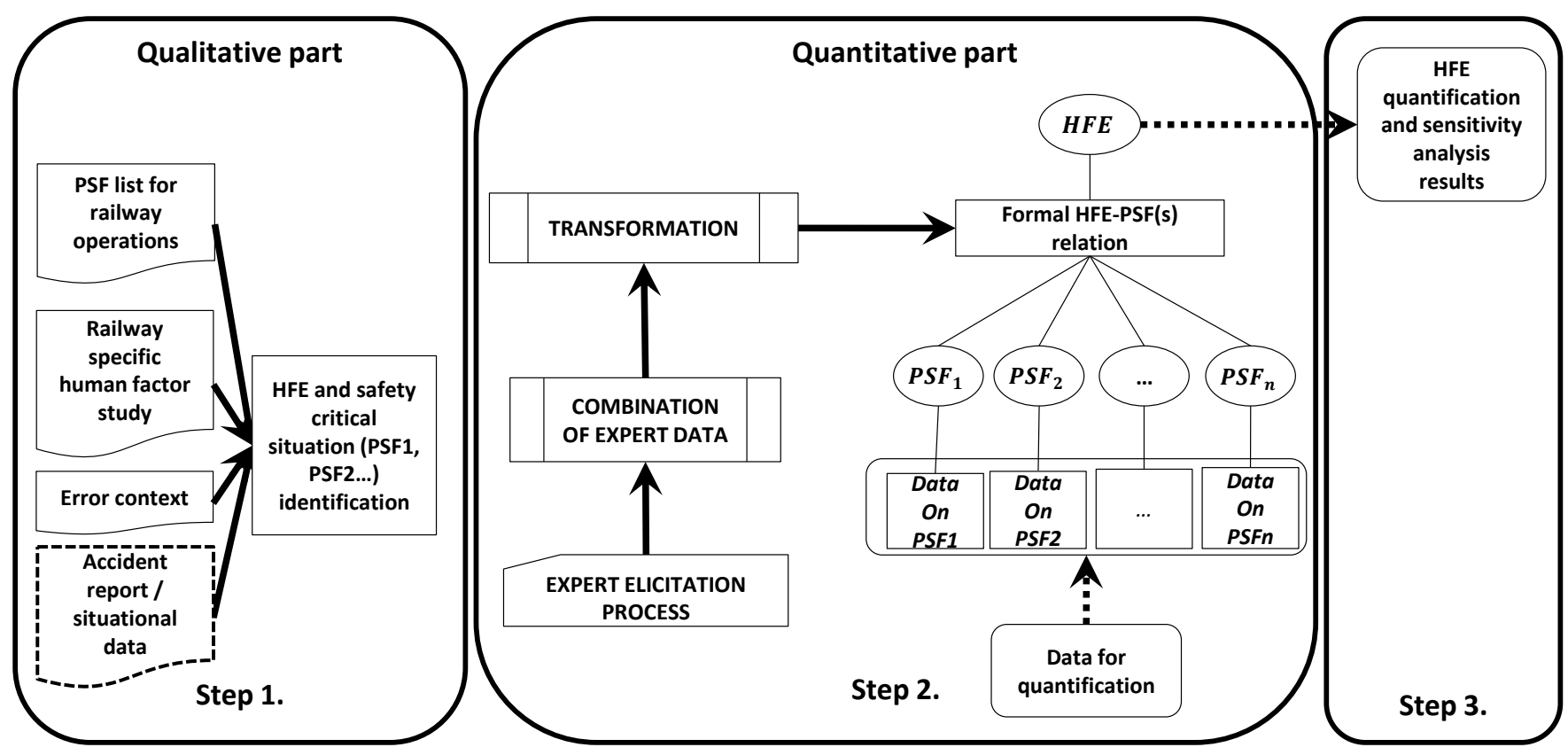

Fig. 1: Overview of the PRELUDE methodology. Step 1. is the qualitative part which aims to identify and characterize a safety critical situation, as HFE and a set of PSFs; Step 2. is the quantitative part, which builds the VBS model from expert data; and Step 3. presents the final HFE quantification and sensitivity analysis results.

\section{The PRELUDE METHOdOLOGY}

PRELUDE an acronym for Performance shaping factor based human Reliability assEssment using vaLUationbaseD systEms, is a human reliability analysis methodology. The complete methodology entails a qualitative part which accounts for human factors and domain specific considerations, a quantitative part which builds an expert system formalizing expert knowledge and providing formal decision-making. To illustrate the proposition, an overview of the PRELUDE methodology is presented in Figure 11. The qualitative and quantitative propositions are detailed in sections 2.2, 2.3 respectively. Finally quantification using a simple example of the obtained VBS model is discussed in section 2.3.4. PRELUDE methodology, as presented in this paper is applied to the railway domain, but it is also adaptable to other applications.

The qualitative proposition of PRELUDE is centered on PSFs and aims for a characterization of a safety critical situation as a set of PSFs. Appropriate rail-specific sources are employed towards concertizing the human factors background of the methodology. The final variable of interest for PRELUDE as with most HRA models is an HFE. HFEs are predefined in terms of disruptions to component, or system functioning, in which humans are involved, either by causing the failure or not preventing or mitigating the failure, and represents the basic unit of analysis in the HRA. It should be remarked that a noticeable difference must be maintained when performing prospective vs. retrospective analysis, since the objective of the analysis changes the variables and their interpretations. This work presents an retrospective analysis approach. However, for a prospective analysis, a discussion to identify HFEs from a human factors study is also presented.

The quantitative part of PRELUDE aims to adequately address all types of uncertainties, especially epistemic, in modeling human reliability and representing expert opinions. Therefore, present work employs VBS and BFT. Thus, before detailing the proposition, the next section presents a background on BFT, combination methods and basic notions of VBS. 


\subsection{Theoretical background}

\subsubsection{Belief Functions Theory and Valuation-based Systems}

Dempster-Shafer theory also known as evidence theory or belief functions theory (BFT) was first proposed in [65] and later extended in [66]. It works with upper and lower probability instead of precise values, with generalization of the Bayesian theory of subjective probabilities. The main contents of this theory are the combination and representation of evidence or knowledge. Evidence can be represented by a basic probability (belief) assignment and distinct pieces of evidence are combined by using a combination rule. It can represent and propagate both (epistemic and aleatory) types of uncertainty. It has been applied to different domains of applications, e.g. data fusion [67], reliability and risk analysis [68] [69], and some aspects of HRA [24].

A model based on BFT can be represented with an Evidential Network (EN). This graphical view offers an easy visualization and usage by non-experts in BFT, and an intuitive display of results. Valuation-Based Systems (VBS) were first defined in [70], and later in [71]. Such ENs present some notable qualities when dealing with uncertainty and decision making [24]. Thus, this framework offers adequate tools to work with uncertainty in data and experts. VBS can also be used to represent several domains for combination of the information viz. Bayesian probability theory, possibility theory, BFT, propositional logic, etc. Present work employs BFT, some of its basic elements are detailed as follows:

Variables and configurations: A finite set of variables are used to model the problem at hand. Let's represent this set of all the variables in the problem by $E=\left\{X_{1}, X_{2}, \ldots \ldots X_{n}\right\}$. For each decision making problem, inference is then drawn only on a reduced domain of interest $\Phi$. For, a variable $X$, its frame $\Omega_{X}$ holds all possible values of this variable, also called as the frame of $X$. Further, for a finite non-empty sub-set of variables $\Phi \subseteq E$, $\Omega_{\Phi}$ denotes the Cartesian product of $\Omega_{\mathrm{Xi}}$ for Xi in $\Phi: \Omega_{\Phi}=\times\left\{\Omega_{X} \mid X \in \Phi\right\}$. Here $\Omega_{\Phi}$ is called the frame (of discernment) for $\Phi$. The elements of $\Omega_{\Phi}$ are considered as configuration of $\Phi$. For example, a set of variables $\Phi=\left\{X_{1}, X_{2}\right\}$, and their respective frames are: $\Omega_{X 1}=\left\{a_{1}, b_{1}\right\}$ and $\Omega_{X 2}=\left\{a_{2}, b_{2}\right\}$, then the frame of discernment for the configuration becomes $\Omega_{\Phi}=\left\{\left(a_{1}, a_{2}\right),\left(a_{1}, b_{2}\right),\left(a_{2}, b_{1}\right),\left(b_{1}, b_{2}\right)\right\}$.

Valuations and Basic Probability assignments (BPA): A valuation $m^{\Omega_{\Phi}}$ holds the knowledge about the possible values of variables in $\Phi$. The set of valuations is denoted by $\Psi_{\Phi}=m^{\Omega_{\Phi}}: \Phi \subseteq E$. A valuation represented by $m^{\Omega_{\Phi}}$ is used to represent knowledge about the possible values (or sets thereof) of $\Phi$. The set of all the valuations in the problem set $\Psi_{E}$ is further divided into two types of valuations: the direct valuations (posterior or input) $\Psi_{D}$ holding the evidence about the input variables; and the prior domain $\Psi_{P}$ holding the valuations that relate the variables amongst themselves. In this work direct valuations are valuations on singletons of $E$ and prior assignments contain at least two elements of $E$. Since, VBS can represent knowledge in different domains; it is possible to express valuations using basic probability assignments (BPAs), possibilities, and so on. Present work uses BPAs, which are an example of a non-zero valuation [72], as described below.

Basic Probability Assignments also known as mass functions are a way of representing confidence in a certain proposition. That is, the confidence that $X$ is equal to a certain numerical or a linguistic value of its frame of discernment. For a variable of interest $X$ and it's frame, $\Omega_{X}$, the mapping $m^{\Omega_{X}}: 2^{\Omega_{X}} \rightarrow[0,1]$ that assigns values to the non-empty sets of the power set $2^{\Omega_{X}}$ in the interval $[0,1]$, is called a basic probability assignment. Further, these mappings are such that for a set $A \in X$ of the power set $2^{\Omega_{X}}: \sum_{A \subseteq \Omega_{X}} m^{\Omega_{X}}(A)=1, m^{\Omega_{X}}(A)>0$ and $m^{\Omega_{X}}(\varnothing)=0$. Here, $A \subseteq \Omega_{X}$ is called a focal set. A $B P A: m^{\Omega}(A)$ hence, reflects the degree of belief (subjective probability) committed to that part of the evidence which exactly points to $A$ and $A$ only. It may be noted that $A$ can either be a singleton (a single value $A=\{a\}$ ) or set of elements $(A=\{a, b\},\{a, b, c, \ldots\})$. A given $B P A$ is similar to a probability function if the focal sets are singletons $(m(A): A=\{a\})$. Further, $B P A m^{\Omega}$ is assigned to each subset of $2^{\Omega}$ instead of $\Omega$, similar as in classical probability theory. Therefore, each focal set has a BPA (strictly positive) based on the evidence about that focal set. Uncertainty about $X$, that is, absolutely no knowledge about the true value of $X$, is represented as the BPA assigned to the whole frame i.e. $\Omega_{X}$. It is represented as $m^{\Omega_{X}}\left(\Omega_{X}\right)$ or simply belief assigned to $\Omega_{X}$. This also extends towards defining relation between two variables (a configuration). For example, let $X$ and $Y$ be two variables with frames $\Omega_{X}=\{a, b\}$ and $\Omega_{Y}=\{c, d\}$ respectively. The relation between $X$ and $Y$ is represented as a joint belief or joint valuation, defined on frame $\Omega_{X Y}=\Omega_{Y} \times \Omega_{X}$ that is $\Omega_{\mathrm{XY}}=\{(a, c),(a, d),(b, c),(b, d)\}[73]$. Here, the joint $B P A$ assignment $m^{\Omega_{\mathrm{XY}}}$ is used to represent the belief about 
the possible relation(s) given by a subset of $2^{\Omega \mathrm{XY}}$, between variables $X$ and $Y$. For example, $m^{\Omega \mathrm{XY}}(\{(a, c)\})=0.8$; $m^{\Omega X Y}(\{(b, c),(b, d)\})=0.2$, are some BPAs representing joint valuations, given that they respect the conditions of its definition. In this work, the term direct belief structure will be used when talking about evidence on single variable $X$, for its frame $\Omega_{X}$. And configuration belief structure for referring to joint valuations which represent relational evidence between two or more variables. That is, for $X$ and $Y$, the $B P A m^{\Omega_{X Y}}$ and corresponding focal sets will be the constituents of a configuration belief structure. This structure formally defines the relation between $X$ and $Y$. Hereafter, these terms focal set and assigned belief or BPA value are used to refer to the contents of a belief structure.

Combination and marginalization: Once the knowledge is represented it has to be combined to make inferences. VBS works on valuations as the objects to be combined or marginalized. A mapping $\oplus: \Psi_{1} \times \Psi_{2} \rightarrow \Psi$ is called combination which aims to combine the knowledge. The combination of multiple valuations: $\oplus \Psi$, is called the joint valuation. As discussed previously, within the framework of BFT there are several combination rules that allow combination of knowledge held by several pieces of evidences. Marginalization is a projection on the frame of the variable of interest (i.e. it aims to crystallize the available combined knowledge on elements of $\Omega_{X}$ for the variable of interest $X$ ). Intuitively, combination corresponds to aggregation of knowledge and marginalization corresponds to crystallization thereof [70]. Under BFT-based usage of VBS, any one of various combination methods can be employed (more in Section 2.1.2). Although Dempster's rule of combination in BFT corresponds to the combination operation in VBS [72] and is what current work employs.

Upper and lower probability bounds: : After performing a combination, the results obtained can be interpreted in the form of a lower $\left(P r_{\text {inf }}\right)$ and upper bound $\left(P r_{\text {sup }}\right)$ or measure on the variable's values. For two subsets $A$ and $B$ of the variable of interest $X$, these are defined as below:

$$
\begin{aligned}
& \operatorname{Pr}_{\text {inf }}(A)=\sum_{B \subset A} m(B) \\
& \operatorname{Pr}_{\text {sup }}(A)=\sum_{B \cap A \neq \varnothing} m(B)
\end{aligned}
$$

Here, the length of the interval (i.e. $\operatorname{Pr}_{\text {sup }}(A)-\operatorname{Pr}_{\text {inf }}(A)$ ) or belief on the frame: $m\left(\Omega_{X}\right)$ represents this imprecision (epistemic uncertainty) about $A$. Finally, suffice to say that the actual probability of $X$ being $A$ is included in the closed interval composed of the lower and upper bounds. Furthermore, $P r_{\text {sup }}>P r_{\text {inf }}$, and if there is an absence of epistemic uncertainty $\operatorname{Pr}_{\text {sup }}(A)=\operatorname{Pr}_{\text {inf }}(A)=\operatorname{Pr}(A)$. Unlike fuzzy set-based approaches probability bounds ease comparison and interpretation of results. Furthermore, it may be noted that all these formulas are compatible with the Bayes rule.

\subsubsection{Combination rules}

Since, this work is also interested in BFT from the perspective of combination of expert data, this section presents a brief description thereof. It may be observed that these combination rules can be used irrespective of the source of data, however, since present work concerns only with experts, the term expert data or opinion is used hereafter. The combination rules and methods employed in present work are briefly given here. Some, well-known methods are: A simple arithmetic average, and a weighted average with specific weighting factors for each expert. These weights can be based on an expert's expertise or domain knowledge and are assigned by an analyst. Through, an independent consensus or vote between the judgments provided by the experts. Methods rooted in BFT can also be used, a more general and exhaustive discussion can be found in [74]. In general, Dempster's rule of combination [65] or product-intersection rule has widespread usage partially because of its ease of application. It considers that the experts are equally reliable and independent. Furthermore, it manages small conflicts by redistributing the conflicting $B P A$ in a uniform way to other focal elements using a normalization factor $1-k$ (where $k$ is a measure of the degree of conflict). Yager's rule [75] was introduced as a modification of Dempster's rule, to address among others, the normalization factor leading to counterintuitive results in cases of highly conflicting evidence. It detects conflict similar to the Dempster's rule, however, it is managed by redistributing the conflicting BPA to the frame of discernment (i.e., it considers conflict as an additional source of uncertainty). To quote [75]: "In this case we 
are saying that since we don't really know anything about the conflicted portion, we let it be distributed among all the elements rather than just those in the focal sets". Suffice to say, there exists plenty of other combination rules such as minimal commitment principle, conjunctive, disjunctive, etc. Each of these rules offer a different set of hypothesis to model and combine knowledge. It may be noted that, when dealing with experts, reliable and independent assumptions are common [76] and easily fulfilled. Furthermore, empirical data, under acceptable assumptions can be also considered independent and reliable.

\subsubsection{Comparison between BPAs using interval and distance metrics}

In the context of belief functions theory comparing given BPAs using a metric such as distance can be useful. This comparison can be used to measure similarity or dissimilarity between the information represented by two BPAs for applications such as clustering, classification, etc. [77]. Such a pairwise comparison can be interesting when BPAs are obtained from different information sources (sensors, experts, etc.) or after using different treatments (such as after combination rules). This distance metric complements the usage of BFT by giving the user a tool to interpret the degree of (non-)alikeness between belief functions in a meaningful way [78]. There are different approaches to compare two BPAs, interested readers can refer to works such as [78] [79].

In the present work's usage of BFT another rather straightforward approach is to use the middle of the interval for a given upper and lower probability bound (also known as the pignistic probability). Such a metric is often used at the decision-making-level in the context of belief functions. In simpler usage it can also be used to compare BPAs, such as the ones obtained after using different combination rules [80]. If different BPAs for a same frame are to be compared, the middle of the interval for a value of interest (e.g. a HFE being true) can be used. It can be limiting in the sense that only a variable's value of interest can be compared across different BPAs.

Secondly, in a more general sense, two BPAs on the same frame can be compared using distance metrics. One of the rather well-known distance measures is Jousselme distance [77]. It proposes the use of a classical similarity measure to achieve the comparison of two BPAs. Let $m 1$ and $m 2$ be two BPAs on the same frame $\Omega$. Then the Jousselme distance $d_{J}$ between $m 1$ and $m 2$ is defined as follows:

$$
d_{J}(m 1, m 2)=\sqrt{\frac{1}{2}\left(\|m 1\|^{2}+\|m 2\|^{2}-2\langle m 1, m 2\rangle\right)}
$$

where $\langle m 1, m 2\rangle$ is the scalar product defined by:

$$
\langle m 1, m 2\rangle=\sum_{i=1}^{n} \sum_{j=1}^{n} m 1\left(A_{i}\right) m 2\left(A_{j}\right) \frac{\left|A_{i} \cap A_{j}\right|}{\left|A_{i} \cup A_{j}\right|}
$$

where $n=\left|2^{\Omega}\right|$ and $A_{i}$ and $A_{j}$ focal sets of all the pieces of information represented by $m 1$ and $m 2$ respectively, and $\|m 1\|^{2}$ the square norm of $m 1$.

As a simpler form of comparing different BPAs for a given variable's value of interest, this paper uses the middle of the intervals. Secondly, the distance metrics presented here $d_{J}$ will also be computed to compare two complete sets of information represented by two given BPAs.

\subsubsection{A simple example}

Current work's usage of aforementioned framework is explained by using a simple example. It is used to describe proposed interpretations of the variables (HFE and PSFs) and valuations (direct and configuration belief structures) in VBS. In a VBS's graphical representation, i.e. a VN, variables are represented by elliptical nodes, and valuations are represented by diamond-shaped nodes, as shown in Figure 2 Here the set of variables of interest are $E=$ $\left\{H F E, P S F_{1}, P S F_{2}\right\}$, where and $H F E$ is the variable of interest and $P S F_{i}$, represents the PSFs.

Their respective frames are defined as $\Omega_{H F E}=\{$ true, false $\}$, and for each of the PSFs as $\Omega_{\mathrm{PSFi}}=\{$ nominal, poor $\}$. The frames are comprised of finite discreet values the variable can take. The relation between the PSFs and HFE is defined by using a configuration belief structure, the BPA represented graphically as $m 1$ in Figure 2 . It is defined on the frame $\Omega_{\Phi}=\Omega_{H F E} \times \Omega_{P S F 1} \times \Omega_{P S F_{2}}$. The other BPAs ( $m 2$ and $m 3$ ) contain evidence on the variables $P S F_{1}$ 


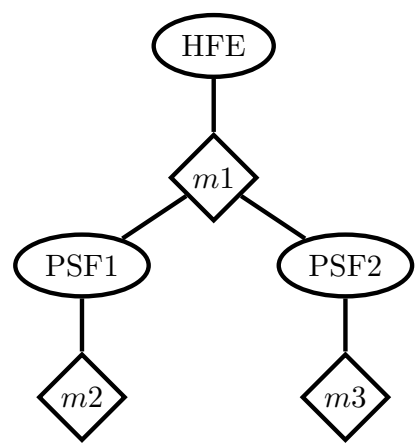

Fig. 2: A simple example and the associated variables modeled as a VBS (a VN).

and $P S F_{2}$ respectively. As discussed before, they are direct belief structures and are used to represent data on the PSF. These direct and configuration belief structures are then used to obtain the quantification results. These quantification results i.e. upper and lower bounds are obtained by a combination of all the BPA and a projection on $\Omega_{\text {HFE }}$. The next sub-sections present the qualitative and quantitative parts of the PRELUDE methodology.

\subsection{Qualitative part}

In some application domains (like railway) a PRA or PRA-like analysis with explicit considerations of HFEs are unavailable. Thus, adequate propositions must be made to extract HFEs and identify safety critical contexts of situations in operational conditions towards HRA considerations. This part describes the identification of the HFEs and characterizes a safety critical situation using PSFs, i.e. variables of the proposed HRA model. In order to represent the domain specific human factor concerns, present work uses a study by the EUAR's human factors network - 'Support Study for Human Factors Integration in European railways' [81] (hereafter referred to as EUAR $H F$ study or simply the study). It presents a detailed analysis of human functions and goals in railway operations in terms of operational safety and other system-level objectives. Also, as of the most recent information from the authors its validation is in progress. The first sub-section presents a generic PSF list for rail operations. In the second sub-section main focus shall be to identify PSFs to characterize safety critical situations in operational conditions.

\subsubsection{Performance Shaping Factors for railway operations}

Generally speaking, PSFs in an HRA should be easy to use and adapted for the application domain. Current work uses a rail-specific PSF list as originally proposed in [82]. It is used in present work as given in Table 2 . Each PSF is accompanied by a definition and a finite number of quantification levels, also known as factor ratings [38] or rating scales or qualitative quality descriptors [4]. The term quantification levels or simply PSF levels are used hereafter. These levels are similar to what is normally seen in other HRA models [62] and activities. This qualitative work considers four levels (good, nominal, poor and insufficient information) for each of the PSFs (Table 2p. These are defined as follows (adapted from [26]):

- Good: A PSF assigned this level is conducive to good performance, such that it reduces the chances for error, and thus, does not pose any safety issues.

- Nominal: It is assigned whenever a PSF is judged to support correct performance, but does not enhance performance (contrary to good) or make tasks easier to carry out than typically expected.

- Poor: A poor level of a PSF is detrimental towards the accomplishment of an objective (leading to the occurrence of a human error).

- Insufficient information: If an expert/analyst does not possess sufficient knowledge to determine whether a PSF can affect a human's performance or if unable to choose among the other alternatives. 
TABLE 2: PSF list with considered definitions and quantification levels, adapted from [82]

\begin{tabular}{|c|c|c|}
\hline $\begin{array}{l}\text { Performance Shap- } \\
\text { ing Factor }\end{array}$ & Definition & $\begin{array}{l}\text { Quantification Lev- } \\
\text { els }\end{array}$ \\
\hline Training & $\begin{array}{l}\text { Have the correct knowledge to perform a job successfully and safely. Training might be needed to } \\
\text { ensure skills are up to date and relevant, i.e., new procedures, different signaling systems, etc. }\end{array}$ & $\begin{array}{l}\text { Good, Nominal, } \\
\text { Poor, Insufficient } \\
\text { Information }\end{array}$ \\
\hline Experience & $\begin{array}{l}\text { The accumulation of information and knowledge gained through interactions with the system and } \\
\text { time spent in the work environment, this can be under conditions (or same route). Aspects like, } \\
\text { bad habits learned, etc. should also be considered in addition to the, positive aspects. }\end{array}$ & $\begin{array}{l}\text { Good, Nominal, } \\
\text { Poor, Insufficient } \\
\text { Information }\end{array}$ \\
\hline Communication & $\begin{array}{l}\text { The ability of team members to pass information to each other and a shared understanding of } \\
\text { the situation using, system status, read-outs, etc. e.g. misunderstanding, omission of, information, } \\
\text { mistaken location, incorrect communication actions. Human-machine communication aspects are } \\
\text { not included in this PSF. }\end{array}$ & $\begin{array}{l}\text { Good, Nominal, } \\
\text { Poor, Insufficient } \\
\text { Information }\end{array}$ \\
\hline $\begin{array}{l}\text { Situational Aware- } \\
\text { ness }\end{array}$ & $\begin{array}{l}\text { The perception of the elements in the environment within a volume of time and space. The } \\
\text { comprehension of their meaning and projection of their status in the near future. As a more general } \\
\text { definition "Skilled behavior, that encompasses the processes by which task-relevant information is } \\
\text { extracted, integrated, assessed, and acted upon." (Endsley 1995). }\end{array}$ & $\begin{array}{l}\text { Good, Nominal, } \\
\text { Poor, Insufficient } \\
\text { Information }\end{array}$ \\
\hline $\begin{array}{l}\text { Time load (Work- } \\
\text { load) }\end{array}$ & $\begin{array}{l}\text { Time required or allocated for one or multiple tasks; this time perception can affect worker stress } \\
\text { beyond the stress of having too many tasks. Available time to complete a task particularly in the } \\
\text { context of driving activities related to high speed trains (both detection and completion of the } \\
\text { task). }\end{array}$ & $\begin{array}{l}\text { Good, Nominal, } \\
\text { Poor, Insufficient } \\
\text { Information }\end{array}$ \\
\hline HSI quality & $\begin{array}{l}\text { An umbrella term to consider the quality of human system interface. The broad context here } \\
\text { includes the procedures, appropriate information displayed to the human at appropriate time or in } \\
\text { an adequate way. It includes most 'Machine-based factors' directly influencing human behavior. }\end{array}$ & $\begin{array}{l}\text { Good, Nominal, } \\
\text { Poor, Insufficient } \\
\text { Information }\end{array}$ \\
\hline
\end{tabular}

PSFs from this list will act as a basis for subsequent identification and characterization of safety critical situations. Other factors will be interpreted as or mapped to PSFs from this list.

\subsubsection{Identification of HFEs and safety critical context}

EUAR HF and similar studies, which provide an operational safety-oriented analysis of human functions, can be used to find detailed analysis on possible safety critical situations. For a prospective analysis, such studies can be used to identify both HFEs and PSFs, whereas for a retrospective analysis the latter is more interesting.

Here, the identification of PSFs from EUAR HF study is explained for one particular human function. For the definitions of terms (italicized) used from the study the reader can refer to the report available on EUAR website (Pickup et al. 2013). Present work considers an HFE as a central starting point of analysis; a safety critical situation is linked to the said HFE in operational conditions. This situation is identified and subsequently represented as set of one or more than one PSFs. For this identification from a human factors study (viz. EUAR HF) a mapping, which functionally matches the HFE to a relevant human function, is needed. Non-accomplishment of this function then represents the HFE. The human functions and its higher level abstraction human function goals can be considered a procedure and a task, respectively. Further, for the objectives of risk analysis and safety in general, only the System purpose/goal of safety (i.e. maintain safety) is considered. Thus, a failure/error in the said human function represents an HFE, at least for the kind of analysis of the current study is concerned. Safety relevant activities and analysis thereof provided in the study consist of discussion on respective conditions (possible scenarios) or casual factors (PSFs) that could possibly lead to errors. To identify an error-causing context (as a set of PSFs) potential for errors and in some cases potential for recovery is used. Current approach only considers explicit statements (or the PSFs stated to be the ones with largest influence) to identify this context. Further, it can be considered 
TABLE 3: Defining Human Failure Type Context for the example HFE

\begin{tabular}{|c|c|c|}
\hline $\begin{array}{l}\text { Failure in Human } \\
\text { function or } \mathrm{HF}\end{array}$ & Performance Shaping Factor identification & $\begin{array}{l}\text { Human Failure Type } \\
\text { Context (HFTC) }\end{array}$ \\
\hline $\begin{array}{l}\text { ERA/HFG5/47: Not } \\
\text { able to route / } \\
\text { re-route passenger or } \\
\text { freight service }\end{array}$ & $\begin{array}{l}\text { Source 1. ERA/HFG5/47: Potential for error - "Trains could be routed towards other } \\
\text { traffic, incompatible infrastructure (59), engineering possessions, or close to } \\
\text { engineering work at high speeds. Errors could be influenced by time pressure or } \\
\text { complexity in track layouts in some situations and locations and problems with } \\
\text { communications (It could be important to consider additional risks at shift } \\
\text { changeover)." } \\
\text { Source 2. PSF list - Training, Experience, Communication, Situational awareness, } \\
\text { Task Load (Workload), Time load (Workload), HSI quality. } \\
\text { Source 3. Error Contexts - EC1 = \{Training, HSI quality, Task load, Communication, } \\
\text { Time load }\}\end{array}$ & $\begin{array}{l}\text { HFTC } C_{H F E}= \\
\{\text { Communication, } \\
\text { Task Load, Time } \\
\text { Load }\}\end{array}$ \\
\hline
\end{tabular}

that the factors with a negative connotation (potential for error, etc.), have a significant effect on the human while performing a said function. This, human function's error-causing factors are then interpreted in terms of PSFs from the PSF list (Table 2). Towards a more robust propositions, error context (ECs) as introduced in [19] are also used.

Since, ECs' application domain is nuclear; the definitions were matched to the PSFs in the PSF list, to keep interpretation of PSFs coherent. The third source is the entire PSFs list, discussed in previous section as a standard base of factors. Common or recurring factors are then selected. The preference in selecting the PSFs in this work such that - EUAR HF study, PSF list and accident/incident report, or safety critical situation/contextual factors identified by experts or analysts - are assigned higher importance than ECs. As evident, a higher preference is assigned to rail domain specific studies. Here, ECs are considered as a characterization of a generic human error causing environment.

Thus, the proposition termed Human Failure Type Context - HFTC, is a qualitative construct which defines a specific HFE (or a safety critical human function) in its safety critical situation. HFTC more generally aims to characterize a safety critical situation as a collection (set) of factors (PSFs) which impede a safe accomplishment of a function by a human, significantly more than individual PSF or other sets thereof. In other words, a simultaneous presence of these factors in a situation will have considerable negative affect on human performance towards the accomplishment of said function. This safety critical situation is represented as a set of PSFs specific to an HFE written as $H F T C_{H F E}$ where $H F T C_{H F E}=\left\{P S F_{i}, P S F_{i+1}, \ldots, P S F_{n}\right\}$.

This approach is illustrated by constructing the HFTC for an example HFE. As discussed before this HFE represents a failure of a human function, for illustration purposes it is taken after being mapped to a human function from a EUAR HF study. Reference to EUAR HF study (more specifically the spreadsheet accompanying it) is given as, ERA/HFGi/j, where $i$ is the index of human function goal and $j$ refers to the index of the specific human function. Non-accomplishment of this human function defines the HFE. Human functional goal ERA/HFG5 states 'To control train movements in all operational circumstances', under which there are multiple (14) human functions. ERA/HFG5/47: Route/re-route passenger or freight service is chosen as the example HFE. Table 3 gives the previously presented process of identifying the constituents of $H F T C_{H F E}$. Common factors (underlined in Table 3 from the three sources are subsequently identified. This gives us $H F T C_{H F E}=$ $\left\{\right.$ Communication,TaskLoad,TimeLoad . Finally, each HFE is modeled as an VBS with its set of PSF $\in H F T C_{H F E}$ in a VBS. This forms the quantitative aspect of the proposed model and is detailed in the next section.

\subsection{Quantitative part}

Quantitative aspect of this proposition concerns with formalizing the evidence(s) to build human reliability model in VBS. Presently, this evidence is obtained from expert elicitations. Information from multiple experts is elicited and combined, followed by a transformation to obtain the final configuration belief structure for the proposed VBS model. 


\subsubsection{The expert elicitation process}

After the variables of the VBS (HFE and its $P S F_{i}: P S F_{i} \in H F T C_{H F E}$ ) are identified and defined, valuations are needed. In this case, these valuations formalize the relation between the HFE and PSFs using configuration belief structures. Towards this objective, a simple question-answer construct is used to capture focused domain knowledge from an expert. In current work it is the occurrence of a human failure (HFE in state true) under specific conditions (characterized by PSFs). Furthermore, the HFE and the PSFs need to be contextualized for the expert. This is accomplished by using information from the EUAR HF study. For a retrospective analysis it can also include comments of investigators, chronology of events, and other data often included in such reports. However, statements which are inferences of the investigators on the factors and HFEs can potentially influence expert beliefs, and thus should be avoided. This additional data hence aims to provide an expert a clear non-ambiguous description of the context.

The current work is only concerned with safety-critical aspects and negative effects of a PSF. Furthermore, explicit considerations of insufficient information are also not employed at present. Thus, only HFE being true and a PSF being poor or nominal are used in the questions. The question is then formulated as conditional on the HFE's occurrence (true) for a poor level of PSF. The text of the question thus forms a proposition and the questionnaire aims to obtain a degree of confidence (as an expert's opinion) on the truthfulness of that proposition. The question for the HFE and the concerned PSF is formulated as: Given the occurrence of a poor level of PSF, what do you think about $H F E$ being true?

The response is expected on a probability scale - number of times out of 10,100,1000, etc., the HFE can said to be true. An expert can use descriptors $-d$ or give directly a subjective probability value. The natural language descriptors or simply descriptors are taken from ATHEANA's elicitation process [20], where similar quantities are elicited. They can however be modified to account for domain specific terminology and/or values. Nevertheless, current work uses them as they are given in [20], where these are defined as follows:

- 'Likely' -0.5 (5 out of 10 would fail)

- 'Infrequently' -0.1 (1 out of 10 would fail)

- 'Unlikely' -0.01 (1 out of 100 would fail)

- 'Extremely unlikely' -0.001 (1 out of 1000 would fail)

- 'Insufficient information'

Thus, the response from the expert takes the form "Given a poor level of a PSF the HFE is true with a probability of $x$ ". Since they are probability values, a response should not exceed unity.

Furthermore, the reasons for eliciting experts for a PSF-HFE pair are as follows:

- A configuration belief structure can represent the conditional relations between multiple variables (PSFs and HFEs) and their values. Therefore, such explicit consideration can be made in the VBS.

- Inter-PSF relations for PSFs internal to a human can be considered generic and sourced from data on human cognition or behavior. For example, a human under extreme task load effecting situational awareness transcends application domain and acceptable generalizations can be made.

- If experts are elicited individually, multi-PSF questions might force them to make conclusions on factors out of their domain expertise. For example, a railway expert might be asked to comment on aspects of human cognition. This limitation is relevant because it is rather common in HRA assessment process to have multiple domain experts each with different expertise.

- Lastly, a HFE-PSF pair presents a generic and simpler context to visualize for the expert, making the elicitation process easier. On the other hand combined effect of multiple PSFs, such as PSF-PSF-HFE, might lead to misunderstanding of the situation and erroneous elicitation.

Hence, the aspects of combinations of PSFs are decoupled from the questions asked to the experts, and put in the core of the quantification model (VBS). Also, since present application is concerned with the true state of an $H F E$ and negative effects of a PSF, a good level is not considered in the questions. However, the model and transformation can account for a good level. This inclusion can be interesting for multiple reasons, accounting 
TABLE 4: The HFE's description and relevant context description and question statements

HFE and questions

ERA/HFG5/47 - Not able to route / re-route passenger or freight service
Context description and question statements

HFE - A signaler was Not able to (Route / re-route passenger or freight service).; Personal and organizational goals - "To respond to scenarios that require trains to be re-routed or travel to a different (unplanned) destination."; Generic context - "Ensure train services can continue operations during engineering / maintenance work, enable engineering trains to get to the work area, provide adequate routing plans, this routing of trains could be planned prior to the work; require short term (re-)planning where there is limited notice of engineering work..."; Potential for error - "Trains could be routed towards other traffic, incompatible infrastructure, engineering possessions, or close to engineering work at high line speeds. Errors could be influenced by time pressure or complexity in track layouts in some situations and locations, and problems with communications (It could be important to consider additional risks at shift changeover)."

Question 1. Task Load - HFE Question 2. Communication HFE

Question 3. Time Load - HFE Question 4. all PSF nominal HFE false
Given the occurrence of a poor level of Task Load, what do you think about HFE being true?

Given the occurrence of a poor level of Communication, what do you think about HFE being true?

Given the occurrence of a poor level of Time Load, what do you think about HFE being true?

Given the occurrence of a nominal level of all the PSFs what do you think about HFE being false?

for the effect of a PSF on suppressing the negative effect of another PSF. Or more generally for a more inclusive human reliability approach. However, this requires some effort on expert elicitation, notably question structure, and combination. On the other hand, some rather intuitive data can be used from human factor studies or in case of empirical data - from simulator experimentation or near miss studies, towards a more inclusive human reliability approach. Nevertheless, it is not considered in the present quantitative proposition.

However, a case representative of normal conditions is added where no human error is considered. This consideration is represented by the question:

"Given the occurrence of a nominal level of all the PSFs what do you think about HFE being false?"

For the HFE and its HFTC obtained after Table 3, questions are given in Table 4. The HFEs and PSFs are contextualized for the expert using data from EUAR HF study, more specifically in section Personal and organizational goals, generic context and potential for error. These choices are facultative and left to the discretion of the analyst. Present work aims at expert independence by eliminating direct expert interaction in the data collection process. For the case study we ensured that there was no interaction among the experts during the expert data collection process, and experts do not have access to each other's responses. And their responses to a question are treated as independent pieces of evidence.

\subsubsection{Combination of expert data}

This section carries forward the discussion on combination rules presented in section 2.1.2. Furthermore, different field of expertise can be useful, especially with a domain such as HRA which touches on multiple domains (reliability, human factors, and application domain-knowledge). However, while mathematically aggregating the data, the hypothesis of the data aggregation method needs to be respected, and the choice remains with the analyst.

Thus, when constructing the quantitative human reliability model, PRELUDE offers to the analyst, a choice of the combination method to use. The choice depends mainly on the hypothesis attached to said rules which can be applied to evidence at-hand. This paper also provides an illustration of what different rules can be used, their hypothesis, and the results. Thus, all combination rules are used in the case study to illustrate some aspects of expert data combination, notably conflict. Some comments are also presented in the case study.

Present work's objective is to understand and demonstrate, when and what method to use, based on the underlying hypothesis. All five of the combination methods are thus used in this paper (ref. section 2.1.2). A summary of their hypothesis and assumptions are given in Table 5.

For a proposition (what the question aims to measure) say ' $X$ is exactly $x$ and only $x$ ' an expert's belief is represented by a $B P A$. The value of this $B P A$, say $b$, is a quantitative expert belief (a subjective probability) on the 
TABLE 5: Combination rules and their hypothesis

\begin{tabular}{|c|c|}
\hline Combination methods & Hypothesis and it's manifestation \\
\hline 1. Arithmetic average & 11 experts are equally reliable. The data received is thus, given equal weight. \\
\hline 2. Weighted average & $\begin{array}{l}\text { A differentiation between experts' domain knowledge is made. This manifests as weights assigned to the } \\
\text { evidence received from each expert. }\end{array}$ \\
\hline $\begin{array}{l}\text { 3. Independent consensus or } \\
\text { majority vote }\end{array}$ & $\begin{array}{l}\text { There is a single correct answer to the question. Therefore, the answer which has the highest frequency } \\
\text { (relative) is chosen. However, if no clear majority amongst the values/descriptors is found, an arithmetic } \\
\text { average is used. }\end{array}$ \\
\hline 4. Dempster's combination rule & $\begin{array}{l}\text { All the experts are equally reliable and evidences are independent. It is associative, commutative but non- } \\
\text { idempotent. It essentially weakens the disagreement and strengthens the agreements in terms of conflict } \\
\text { in the elicited values. }\end{array}$ \\
\hline 5. Yager's rule [75] & $\begin{array}{l}\text { This rule assumes that all the experts are reliable and the evidences are independent. It is quasi-associative, } \\
\text { commutative but not idempotent. Contrary to previous case the conflict manifests itself as uncertainty. }\end{array}$ \\
\hline
\end{tabular}

said proposition. Each expert's response is then modeled as a complementary belief structure. This goes to state that, for each expert, belief about the value of $X$ being $x$ is $b$ and exactly $b$. Therefore, the belief of $X=\{\bar{x}\}$ is $1-b$. This is then modeled as two focal sets with the associated BPA values. The belief structure in 4 gives the considered representation of expert data.

$$
\begin{aligned}
& m(\{x\})=b \\
& m(\{\bar{x}\})=1-b \\
& m\left(\Omega_{X}\right)=m(\{x, \bar{x}\})=0
\end{aligned}
$$

Finally, a single response (a quantitative value) for each question (PSF-HFE pair) is obtained; this is used in the next section to complete the VBS model construction.

\subsubsection{Transformation}

Combination of data gives a single piece of evidence per PSF-HFE pair. This evidence is a combined quantitative measure of the experts' opinion in each question's proposition. These measures and propositions are used for constructing the configuration belief structure for the VBS model. Towards this objective, a transformation is to define the final configuration belief structure of the VBS model.

First, each question's proposition represents a conditional piece of evidence, viz. a conditional belief of a state of an HFE given a state of a PSF. This conditional belief must be deconditioned to accommodate it in the dynamic part of VBS. The relations between variables should be transformed to valuations or joint belief. Thus, Smet's rule [73] is employed, it proposes a transformation of a conditional piece of evidence into a joint belief structure. It represents a relation between two variables $\mathrm{A}$ and $\mathrm{B}$, defined as given in equation 5 .

Given the conditional evidence if $A=a$ then $B=b$ with a $B P A=x$.

The rule is represented by a belief structure defined on $E: \Omega_{E}=\Omega_{A} \times \Omega_{B}$, such that:

the focal set $\left\{(a, b)(\bar{a}, b)(\bar{a}, \bar{b}\}\right.$ is assigned a $B P A=x$ and the focal set $\Omega_{E}$ is assigned a $B P A=1-x$

After deconditioning, using the rule given in equation 5 , for every question, an initial belief structure is obtained which relates a particular PFS and a HFE with a $B P A$ value. This initial belief structure is defined on the frame $\Omega_{\Phi}=\Omega_{\mathrm{PSFi}} \times \Omega_{\mathrm{HFE}}$.

Second, $H F E=\{($ true $)\}$ and $P S F=\{($ poor $)\}$ is the minimal explicit information in a question's proposition. On the other hand, the VBS model quantifies using a set of valuations. For present work this valuation (the configuration belief structure) relates $\mathrm{HFE}$ and all the PSF $\in H F T C_{\mathrm{HFE}}$. That is, it reasons with the HFE and with all the PSFs in an given safety critical situation (i.e. $H F T C_{H F E}$ ). Effectively it is defined on the frame $\Phi: \Omega_{\Phi}=\left\{\Omega_{H F E} \times \Omega_{\mathrm{PSFi}} \mid \forall\right.$ PSFi $\left.\in H F T C_{H F E}\right\}$, i.e. all the states of all the PSFs in an HFTC and the relevant 
HFE. Hence, a transformation is needed to obtain the complete final configuration belief structure. Thus, a vacuous extension is performed on the initial belief structure (if needed). This gives the intermediate belief structures for each question. The second part of this transformation combines all the questions' propositions, represented as their respective intermediate belief structures to obtain the final configuration belief structure. This transformation, thus entails a vacuous extension of a question's initial belief structure giving the intermediate belief structure and finally the combination thereof to obtain the final configuration belief structure.

This transformation is explained by using the HFE's questions given in Table 4. It is considered that after combination (ref. section 2.3.2) the final probabilistic responses are obtained as follows:

- Combined probabilistic response for Question 1. : 0.05

- Combined probabilistic response for Question 2. : 0.2

- Combined probabilistic response for Question 3. : 0.001

- Combined probabilistic response for Question 4. : 0.95

Question 1 from Table 4 can be written as: if Task Load $=\{($ poor $)\}$ then $H F E=\{($ true $)\}$, with the combined probabilistic response representing the quantitative measure of evidence on the truthfulness of said proposition, here it is 0.05 . This proposition is deconditioned using the rule in equation 5 giving two initial belief structures (BPAs):

$$
\begin{gathered}
m Q 1^{\Omega_{H F E} \times \Omega_{T a L}}(\{(\text { poor }, \text { true })(\overline{\text { poor }}, \text { true })(\overline{\text { poor }}, \overline{\text { true }})\}) \\
=m Q 1^{\Omega_{H F E} \times \Omega_{T a L}(\{(\text { poor }, \text { true })(\text { nominal }, \text { true })(\text { nominal }, \text { false })\})}=0.05, \\
\text { and } \\
m Q 1^{\Omega_{H F E} \times \Omega_{T a L}\left(\Omega_{H F E} \times \Omega_{T a L}\right)} \\
=m Q 1^{\Omega_{H F E} \times \Omega_{T a L}}(\{(\text { poor }, \text { true })(\text { nominal }, \text { true })(\text { poor }, \text { false })(\text { nominal }, \text { false })\})=0.95
\end{gathered}
$$

Similarly initial belief structures can be obtained for Questions 2 and Question 3. Each of these initial belief structures contain two elements per set. Note that, Questions 4 however, represents a relation between all the PSFs and the HFE. It is therefore interpreted as: if (TaskLoad, Communication, TimeLoad $)=\{($ nominal, nominal,nominal $)\}$ then $H F E=\{($ false $)\}$. The initial belief structure thus in this case contains four elements per set. Thus, all of the focal sets using equation 6 and combined probabilistic responses as the BPA's are given below. These initial belief structures (that is the respective focal sets and BPAs) for the questions obtained after deconditioning using equation [73], are given below:

Question 1. represented as $\mathrm{mQ} 1^{\Omega_{\mathrm{HFE}}} \times \Omega_{\mathrm{TaL}}$, which gives first focal set as: $\{($ poor, true) (nominal, true) (nominal, false) $\}$, with a $B P A=0.05$; and second focal set as: $\left\{\Omega_{\mathrm{HFE}} \times \Omega_{\mathrm{TaL}}\right\}=\{($ poor, true $)($ nominal, true $)($ poor, false) $($ nominal, false $)\}$ with a $B P A=0.95$.

Question 2. represented as $\mathrm{mQ} 2^{\Omega_{\mathrm{HFE}} \times \Omega_{C}}$ which gives first focal set as: $\{$ (poor, true) (nominal, true) (nominal, false) $\}$ with a $B P A=0.2$; and second focal set as: $\left\{\Omega_{\mathrm{HFE}} \times \Omega_{C}\right\}=\{($ poor, true $)($ nominal, true $)($ poor, false $)($ nominal, false $)\}$ with a $B P A=0.8$.

Question 3. represented as $\mathrm{mQ} 3^{\Omega_{\mathrm{HFE}} \times \Omega_{\mathrm{TiL}}}$ which gives first focal set as: $\{($ poor, true) (nominal, true) (nominal, false) $\}$ with a $B P A=0.001$; and second focal set as: $\left\{\Omega_{\mathrm{HFE}} \times \Omega_{\mathrm{TiL}}\right\}=\{($ poor, true $)($ nominal, true $)($ poor, false $)($ nominal, false $)\}$ with a $B P A=0.999$.

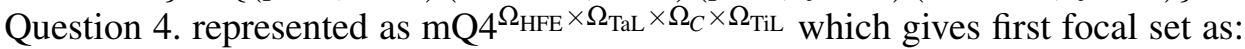
$\{$ (nominal, nominal, nominal, false),(poor, poor, poor, false), (poor, poor, poor, true) $\}$ with a $B P A=0.95$ and second focal set as:

$\{$ (nominal, nominal, nominal, false),(poor, poor, poor, false) (nominal, nominal, nominal, false),(poor, poor, poor, true) $\}$ with a $B P A=0.05$.

As can be seen in the first column of above equations questions 1,2 and 3 are defined on $\Omega_{\mathrm{HFE}} \times \Omega_{\mathrm{TaL}}$, $\Omega_{\mathrm{HFE}} \times \Omega_{C}$ and $\Omega_{\mathrm{HFE}} \times \Omega_{\mathrm{TiL}}$ respectively. Thus, these initial belief structures need vacuous extension. However, Question 4 is already defined on the frame $\Omega_{\mathrm{HFE}} \times \Omega_{\mathrm{TaL}} \times \Omega_{C} \times \Omega_{\mathrm{TiL}}$. It thus, does not need a vacuous extension. Thus, a vacuous extension is performed for questions 1,2 and 3. This process is detailed below: 
For Question 1's proposition the obtained $B P A \mathrm{mQ1} \Omega_{H F E} \times \Omega_{T a L}$ is defined on the frame $\left\{\Omega_{\mathrm{TaL}} \times \Omega_{H F E}\right\}$, whereas in the present VBS model, the configuration belief structure is defined on the frame $\Omega_{H F E} \times \Omega_{T a L} \times$ $\Omega_{C} \times \Omega_{T i L}$. Thus, a vacuous extension is performed by doing a cross product of the focal set of the initial belief structures, and frame of Task load and Communication, this extension is given as follows:

$$
\begin{aligned}
& m Q 1^{\Omega_{H F E} \times \Omega_{T a L}}(\{(\text { poor }, \text { true })(\text { nominal }, \text { true })(\text { nominal }, \text { false })\}) \\
& =m Q 1^{\Omega_{H F E} \times \Omega_{T a L} \times \Omega_{C} \times \Omega_{T i L}(A)} \\
& \text { where } \\
& A=\{(\text { poor }, \text { true })(\text { nominal }, \text { true })(\text { nominal }, \text { false })\} \times\left\{\Omega_{C} \times \Omega_{T i L}\right\} \\
& =\{(\text { poor }, \text { true })(\text { nominal }, \text { true })(\text { nominal }, \text { false })\}) \times\{(\text { poor }, \text { nominal })\} \times\{(\text { poor }, \text { nominal })\} \\
& =\{(\text { poor }, \text { true }, \text { poor }, \text { poor })(\text { poor }, \text { true }, \text { poor }, \text { nominal })(\text { poor }, \text { true }, \text { nominal }, \text { poor }) \ldots\}
\end{aligned}
$$

Here, $A$ is the focal set for the BPA $m Q 1^{\Omega_{H F E} \times \Omega_{T a L} \times \Omega_{C} \times \Omega_{T i L}}$. The complete focal set thus obtained is defined on the frame $\Omega_{H F E} \times \Omega_{T a L} \times \Omega_{C} \times \Omega_{T i L}$. It thus, contains four elements per set, instead of previous two. Similarly, equation 7 is repeated for all of the questions' focal sets, with appropriate frames to obtain intermediate belief structures for each of them. After obtaining intermediate belief structure, for each question defined on the same frame, a combination can be performed. This gives a single piece of relational evidence from all of the questions for an HFE. The independence constraints are respected because the responses are independent of each other. Hence, they can be combined using Dempster's rule as given in equation 8 .

$$
\begin{array}{r}
m 1=m Q 1^{\Omega_{H F E} \times \Omega_{T a L} \times \Omega_{C} \times \Omega_{T i L}} \oplus m Q 2^{\Omega_{H F E} \times \Omega_{T a L} \times \Omega_{C} \times \Omega_{T i L}} \oplus m Q 3^{\Omega_{H F E} \times \Omega_{T a L} \times \Omega_{C} \times \Omega_{T i L}} \oplus \\
m Q 4^{\Omega_{H F E} \times \Omega_{T a L} \times \Omega_{C} \times \Omega_{T i L}}
\end{array}
$$

This gives us the final configuration belief structure concluding the construction of the VBS model, from the the simple questions (section 2.3.1). More generally, multiple PSFs and an HFE (PSF-PSF-... -HFE ) questions are not asked from the experts in present work, they can very well be implemented in the current proposed approach (by adequately changing the vacuous extension). Nevertheless, in our configuration belief structure there is always a component of an HFE. Pure PSF-PSF are currently not considered. However, mathematically speaking, as is the case with the work discussed before [19], a PSFs influence on another PSF can be modeled using an intermediate belief structure, which, for example can be a belief structure between Task Load and Communication; which then links to the central configuration belief structure.

\subsubsection{Example HFE's quantification}

For the HFE from Table 3, the domain of interest is $\Phi=\{H F E, T a L, C, T i L\}$. Here, HFE is the HFE under analysis, and the PSFs are Task Load (TaL), Communication $(C)$ and Time Load (TiL). Their respective frames are defined as $\Omega_{\mathrm{HFE}}=\{$ true,false $\}$, and for each of the PSFs as $\Omega_{\mathrm{PSFi}}=\{$ nominal,poor $\}$. The relation between the PSFs and HFEs, is defined by the configuration belief structure obtained after equation 8 , represented graphically as $m 1$ in Figure 3

Here, $m 1$ is defined on the frame $\Omega_{\Phi}=\Omega_{H F E} \times \Omega_{T a L} \times \Omega_{C} \times \Omega_{T i L}$. The other BPAs $m 2, m 3$ and $m 4$ contain evidence on the variables $T a L, C$ and $T i L$ respectively. As discussed before, they are direct belief structures and are used to represent data on the PSFs.

Data for quantification: PRELUDE's VBS model takes data on the PSF(s) to quantify the HFE (the variable of interest). This data is represented in the direct belief structure(s). It can come from a given operational or an accident context, and is assigned by an analyst. It simplifies the usage and eliminates the aspects of subjectivity on the choice of PSFs and their affects (given the VBS model is constructed beforehand) by an analyst during the analysis process.

Since, current application deals with a retrospective analysis, this evidence is obtained from relevant accident analysis statistics. For present work [52] is chosen for its relevance to the domain and availability of data matching the current need. That is the number of times a PSF was one of the causal factor(s) given there was a human 


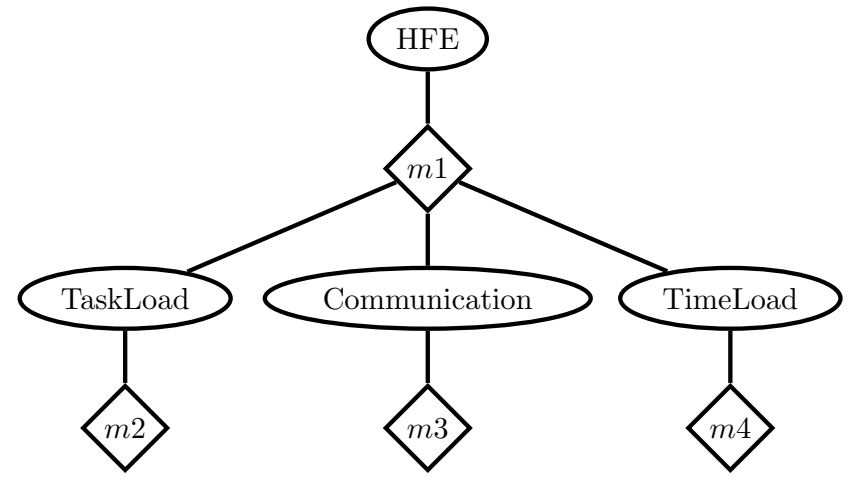

Fig. 3: The example HFE and related PSFs modeled as a VBS.

TABLE 6: Direct belief structures for HFE: from the R-PSF equivalent as identified focal sets and associated $B P A$ values

\begin{tabular}{lllll}
\hline Cause R-PSF from $|83|$ & $\begin{array}{l}\text { Identification frequency vs. } \\
\text { Total number of accidents }- \\
d a_{P S F}\end{array}$ & $\begin{array}{l}\text { PSF from Table 1 and rep- } \\
\text { resentation in Figure 3 }\end{array}$ & $\begin{array}{l}m^{P S F i}(\{(\text { poor })\}) \\
d a_{P S F}\end{array}$ & $\begin{array}{l}m^{P S F i}(\{(\text { nominal })\})= \\
1-d a_{P S F}\end{array}$ \\
\hline $\begin{array}{l}\text { Workload, Time pres- } \\
\text { sure, Stress }\end{array}$ & $58 / 167 \oint^{1}=0.0173$ & Task Load $(m 2)$ & 0.0346 & 0.965 \\
$\begin{array}{l}\text { Communication, Team- } \\
\text { work }\end{array}$ & $228 / 1676=0.136$ & Communication $(m 3)$ & 0.136 & 0.864 \\
$\begin{array}{l}\text { Workload, Time pres- } \\
\text { sure, Stress }\end{array}$ & $58 / 1676=0.0173$ & Time Load $(m 4)$ & 0.0346 & 0.965 \\
\hline
\end{tabular}

involvement in an event (accident, severe accident, etc.). Furthermore, if a PSF is identified as a cause of an accident, it can be safely assumed to be in a poor state, or a state which degrades human performance in general. Also, they arrive at R-PSF and their occurrence frequencies after merging the PSFs (and subsequently their occurrence frequencies) in multiple steps (definitions, categorization, and threshold). That is, we can consider that after these multiple levels of combinations, the resulting PSFs and their frequencies are sufficiently independent, to be considered as such in our model.

Thus, in present case a direct belief structure represents proposition that a PSF level is (\{poor $\})$. The quantitative measure on this evidence is obtained as: number of times a PSF is reported to be a direct cause vs. total number of events. Thus, the proposition is represented by the focal set $\{($ poor $)\}$ and the quantitative measure on this evidence assigns the BPA: $d a_{P S F}$ (for direct assignment to a PSF). If PSF was not in a poor state, it is everything except poor. That means, it is nominal, since for current work $\Omega_{P S F i}=\{($ nominal,poor $)\}$ such approximations are sufficiently conservative for present work. This gives us the proposition and evidence thereof as $m^{P S F}(\{($ nominal $)\})=1-d a_{P S F}$. Similarly for all the PSFs, and thus, direct belief structures for the VBS model are obtained (refer to $m 2, m 3, m 4$ in Figure 3). The direct evidences come from independent and reliable sources. That is, all the evidence obtained from accidents are independent ( 1.5 PSFs identified per accident report) and reliable (accident/incident investigation reports) [52]. Thus, in this case Dempster's rule can be employed.

2.3.4.1 Quantification and sensitivity analysis results: Figure 4 shows the graphical model of the implementation for quantification of HFE (after representation in Figure 3) in VBS, with the direct and configuration belief structures titled what they contain. The direct assignment for Task Load is represented by the diamond shaped node - DataOnTaL. It contains two focal sets $\{$ nominal $\}$ and $\{$ poor $\}$, and respective BPA values as $m^{\mathrm{TaL}}(\{$ nominal $\})$ and $m^{\mathrm{TaL}}(\{$ poor $\})$, the latter obtained from accident statistics, as discussed in the previous section. Similarly for Time Load and Communication, the direct assignments are given in Table 6 
TABLE 7: Marginalization results for example HFE on $\Omega_{H F E}$

\begin{tabular}{ll}
\hline Values of the example HFE & Measure on the HFE's values obtained after marginalization \\
\hline true $\}$ & 0.00005 \\
$\{$ false $\}$ & 0.94981 \\
$\Omega_{\mathrm{HFE}}=\{$ true, false $\}$ & 0.05014 \\
\hline
\end{tabular}

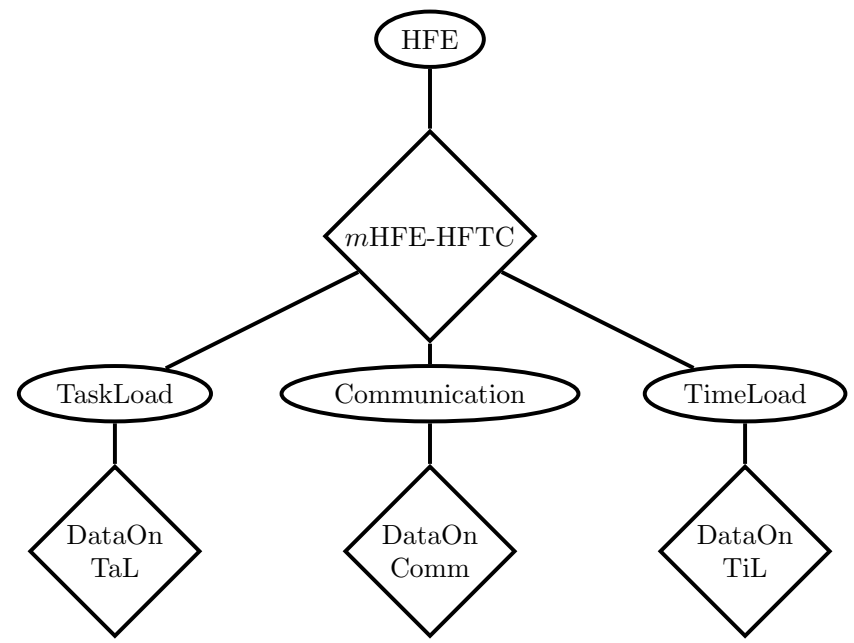

Fig. 4: Implementation in VBS for the quantification of HFE.

After defining all the direct belief structures, BFM (Belief Functions Machine) [84] is used to combine the direct and configuration belief structures and marginalize for HFE. The results obtained by marginalization, i.e. projection on $\Omega_{H F E}$ are given in Table 7 . The obtained results are represented as upper and lower probability bounds (equation 11. Since, human reliability analysis and also present work is concerned with an HFE being true these quantification results are represented in the form of an interval, given as: $\left[\operatorname{Pr}_{\text {inf }}\left(\operatorname{HFE}\left(\right.\right.\right.$ true $\left.\left._{)}\right), \operatorname{Pr}_{\text {sup }}(\operatorname{HFE}(\operatorname{true}))\right]=$ $[0.00005,0.05019]$

As a natural second step, a feedback for the analysis undertaken by the analysts can be performed. Such a feedback aids decision making by allowing an analyst to perform a diagnosis on the individual PSFs, such an activity can be interpreted as suggestions for improvements to be made to reduce HEP. In PRELUDE's context sensitivity analysis aims to give a formal feedback towards safety objectives. It ranks PSFs in terms of their contribution towards the variable of interest (HFE). This makes it possible to establish a priority ranking, towards improvements in PSFs needed for effective gains in operational safety and to identify PSFs on the other end of this list, which can be ignored.

In VBS terminology this step is known as a reverse propagation. The VBS model is modified as follows: given the occurrence of the HFE (HFE is true, in present case), marginalize for every PSF (poor state) in the HFTC $C_{H F E}$. An HFE is assigned a direct belief structure as: the focal set of $\{($ true $)\}$ with a BPA value equal to 1, and marginal on all the other variable's (PSFs) states is calculated. The obtained marginal for each PSF is combined with the direct evidence thereof (again using Dempster's rule). It is essentially an updating of evidences, a combination of a prior (obtained from experts - the configuration belief structure and the HFE being true) and a posterior (assigned for the application, represented by direct belief structure as obtained in section 2.3.4 evidence. Finally, towards safety objectives only the state of PSF under analysis is kept (i.e. poor), other focal sets and their BPAs are not discussed. The results of the sensitivity analysis are presented as a bar graph. From the marginal obtained for the poor state of a PSF, relative values are calculated and presented in percentage form. In other words each bar represents the relative percentage contribution of a PSF being poor leading to the HFE being true. This is 


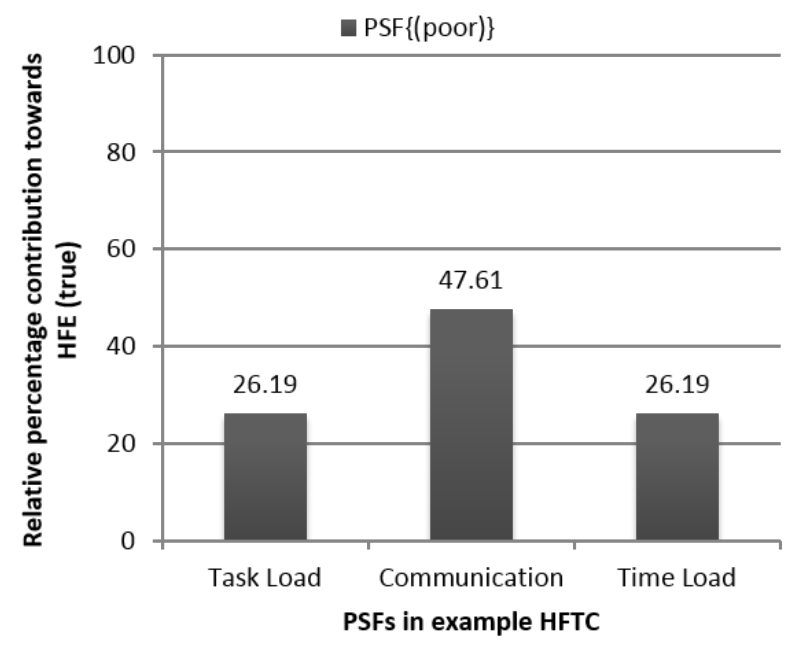

Fig. 5: Sensitivity analysis results for example HFE and associated PSFs.

interpreted as the contribution of a PSF towards causing an 'error'. It is to be noted, that this choice of states of the variables (poor and true) is driven by the objectives of the current analysis. A reverse propagation can be performed on any variable and any of its state(s). The results obtained for the example HFE are shown in Figure 5. It can be seen that Communication has clearly a higher contribution than the other two PSFs. Thus it can be concluded that improving the aspects of Communication should be the priority.

It can be remarked here that for questions of the example HFE, expert valuations ( $b p a$ values) are different (i.e. combined probabilistic response for a question, as given in section 2.3.3). On the other hand, the direct belief structures in Table 6 are the same for the case of Time Load and Task Load. Now, as seen in Figure 5 s, the feedback seems to reflect direct evidence, as it states Task Load and Time Load (for their poor level) to be equally likely contributors towards the HFE. However, it does not seem to reflect the differing expert valuations. This is due to the fact that the configurations belief structure's focal set is symmetric, as obtained by the transformation approach from equation 8 . This leads to the marginal obtained in the first step of sensitivity analysis being same across all PSFs. This is a constraint stemming from the simpler questions asked to the experts, as a question with more than one PSFs can have a non-symmetric focal set in the configuration belief structure. But then again an expert might find it difficult to respond to such questions, however, other data sources (such as simulator experimentation) can be used. This concludes the quantitative proposition employing the VBS model.

\section{CASE Study}

This section presents the application of PRELUDE on a recent catastrophic high-speed railway accident's scenario. In this accident human error was concluded to be as one of the primary causes. Data (factors, events, etc.) are taken from the official investigation report [85]. This work neither aims to nor can achieve the detailed and exhaustive analysis provided in the official investigation report. Here, the prime motive is to demonstrate the usage and application of proposition as a retrospective analyses.

The usage of PRELUDE in this case study is demonstrated by employing the three steps as shown in Figure 1. This application process is generic; the way in which each step is conducted dependents upon the purpose of the analysis. Step 1 follows the traditional sense of defining the scope of the analysis, and analyzing an accident scenario to identify the HFEs and related PSFs, to characterize a safety critical situation. For a prospective approach this can be a procedure and operational context. Step 2 puts PRELUDE's quantitative propositions from section 2.2 and section 2.3 into action - elicitation of data from experts, and combination and transformation thereof. Finally, in Step 3, the quantification data for application (direct evidence) is entered in the model, and the results of quantification and sensitivity analysis are presented and commented on. 
TABLE 8: HFE and relevant procedures from the accident investigation report and national regulations

Identified Human Failure Event

from the accident report

HFE1. Ineffective

communication

HFE2. Not respecting the speed signals (in schedule book/table of speeds)

HFE3. Not reducing speed in time
Source and procedure/task description

Accident investigation report - “. . . a contributing cause was an absence of attention of the train driver ... after answering a phone call..." No strict procedures/rules (except a guide to good practice in driving.)

Accident investigation report - “... not respecting the prescribed maximum speed allowable by infrastructure, as established in the tables of maximum speeds mentioned in the train Schedule book..."

Accident investigation report - "driver should identify the reference (point) to initiate the braking and to reduce the speed." Regulations - "The driver shall endeavor to recognize the signs (signals) as far as possible and do not lose interest in their observation as (long as) it (train) has not crossed them."

\subsection{Step 1. Qualitative part: HFE and PSF(s) identification}

Before the application of PRELUDE, this step defines scope of the analysis to limit the problem-set. Main considerations include: type (retrospective), and detail (procedures, and human actions or functions). Since current scope is limited to analyzing the accident scenario and demonstrating key aspects of the proposition, thus, only the immediate HFEs and PSFs which are direct causes of the accident are analyzed.

The report provides a detailed and chronological account of noteworthy events which led to the accident in question. It is thus, used to identify the HFEs. A reverse task-analysis approach is implemented, where the starting point is the immediate safety critical events involving a human (HFEs) in the accident report. Further, as needed detailed operating procedures and the signaling principles were consulted from the national regulation documents, such as directive guidelines which contain procedures requiring 'passive and immediate obedience' from a human actor. These directives can be considered to have a higher priority than for example, non-regulatory or non-normative guidelines such as 'good practices in driving'. The sources and how they are used is given as follows:

- Accident investigation report - identification of HFE (non-accomplishment of a task or function) and description.

- National regulations - detailed description of the procedures, previously identified task is part of.

- Human factors studies (EUAR HF) - identification of safety critical situation mapping of human function and previously identified HFE.

Finally, the identified HFE, excerpts from the report and the relevant procedures given in Table 8 .

The plot in Figure 6 aims to show the HFEs (annotated in yellow-red ovals) in chronological occurrence of the accident scenario. The horizontal axis represents distance from the accident point in meters and vertical axis represents speed in $\mathrm{km} / \mathrm{h}$.

Further, the safety critical situation of the respective HFEs needs to be identified. This activity follows steps of section 2.2 identify the PSFs and subsequently $H F T C_{H F E}$ for every HFE. The EUAR HF study is also used here, for additional identification of the PSFs, using a mapping of HFEs to human function. A human function which matches closest to the HFE under analysis is identified from the EUAR HF study. EUAR/HFG4/35 is defined as "Maintain appropriate speed" which entails the personal and organizational goal - "To ensure movements at a speed that is safe for the vehicle in the current conditions and in accordance with the timetable." This definition matches with both the HFE2: Not respecting the speed signals in schedule book) and HFE3: Not reducing speed in time, as identified in Table 8. In addition to the sources used in section 2.2.2 (i.e. EUAR HF study, PSF list, ECs) extracts from accident investigation report are also used to account for accident scenario specific PSFs. It gives a retrospective account of casual factors that influenced the human towards those errors. For example, it states - 'the train driver (the human actor) did not brake (the activity required of him) because of distraction (the 


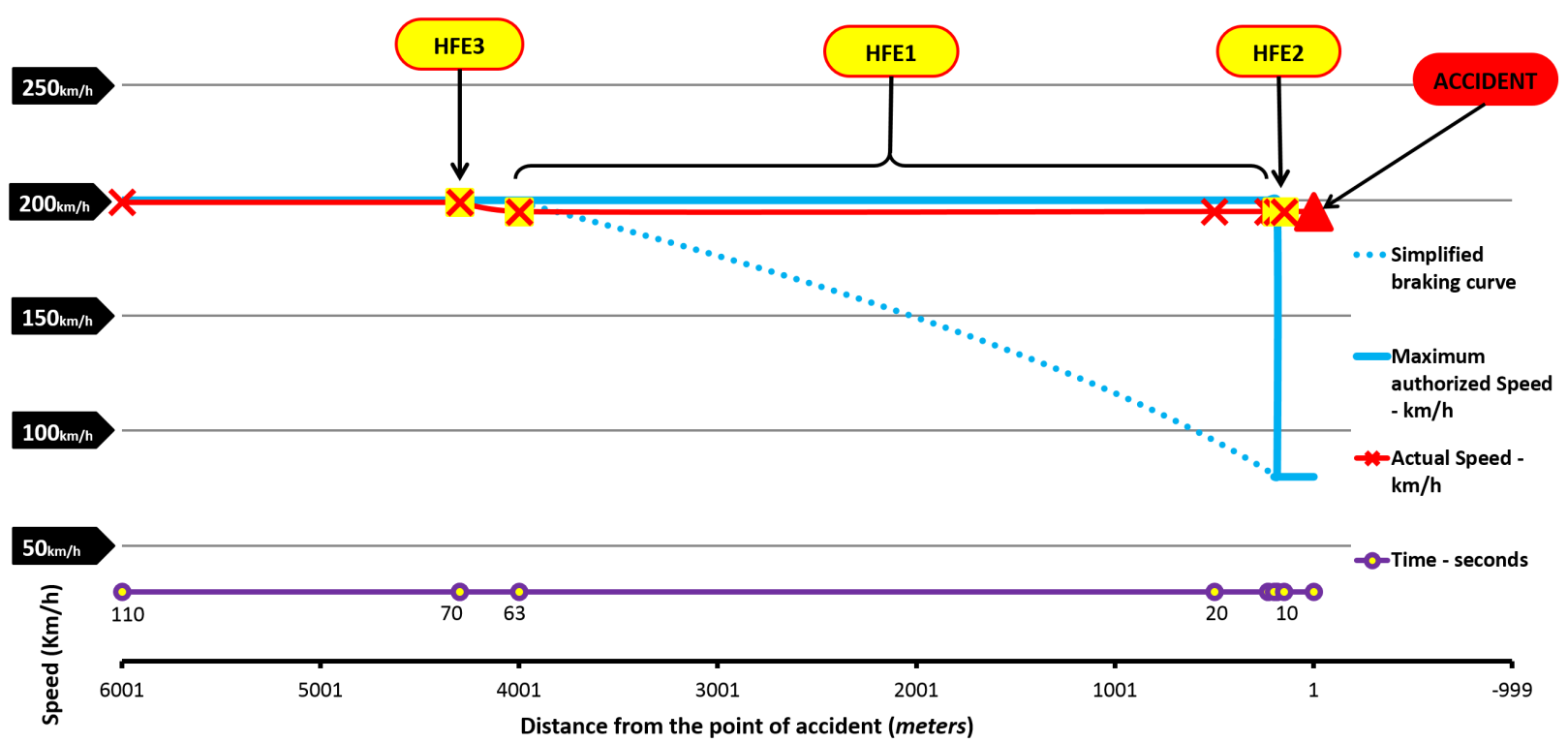

Fig. 6: Accident scenario: speed of the train vs. distance from the point of accident. The data points (cross marks) are other events as identified in the investigation report and the HFEs. Also a time scale is given to represent the time duration of the analyzed scenario.

factor)'. Hence, the context is dealing with the train driver performing the human function/task of braking; the act of not braking (non-accomplishment) is the HFE. Here, 'distracted' becomes a factor of interest for a human function's error (HFE) context $H F T C_{H F E}$. It is important to note that, the use of accident report augments the HFTC's construction and is application specific (qualitative part, dashed document box Figure 1). All the identified PSFs and their sources, and the recurring PSFs (underlined based on the preference presented in section 2.2.2), and finally the HFTCs are given in Table 9.

TABLE 9: Identification of PSFs for defining HFTC for HFEs from the accident scenario

\begin{tabular}{|c|c|c|}
\hline $\begin{array}{l}\text { Human } \\
\text { Failure } \\
\text { Event }\end{array}$ & Performance Shaping Factor identification & $\begin{array}{l}\text { Human Failure Type } \\
\text { Context }\left(H F T C_{H F E}\right)\end{array}$ \\
\hline HFE1 & $\begin{array}{l}\text { EUAR/HFG5/53 - Communication; PSF list }- \text { Experience, Communication, Situational } \\
\text { awareness, Task Load (Workload), Time load (Workload), HSI quality; Accident } \\
\text { investigation report }- \text { Communication; Error Context }- \text { EC1 }=\{\text { Training, HSI quality, } \\
\text { Task load, Communication, Time load }\}\end{array}$ & $\begin{array}{l}H F T C_{H F E 1}= \\
\{\text { Communication }\}\end{array}$ \\
\hline HFE2 & $\begin{array}{l}\text { EUAR/HFG4/35 - Training (skill), Experience (local knowledge); PSF list - Training, } \\
\text { Experience, Communication, Situational awareness, Task Load (Workload), Time load } \\
\text { (Workload), HSI quality; Accident investigation report }-\underline{\text { Situational Awareness }} \text { ('lack } \\
\text { of attention'), HSI quality ('lack of regulation on track-side fixed preventive signaling'); } \\
\text { Error Context }-\mathbf{E C 2}=\{\underline{\text { Situational awareness, Experience }\}, \mathbf{E C 3}=\{\text { Task load, }} \\
\text { HSI quality, Time load, } \underline{\text { Situational Awareness }}\}\end{array}$ & $\begin{array}{l}H F T C_{H F E 2}= \\
\{\text { Experience, Situational } \\
\text { Awareness, HSI quality }\}\end{array}$ \\
\hline HFE3 & 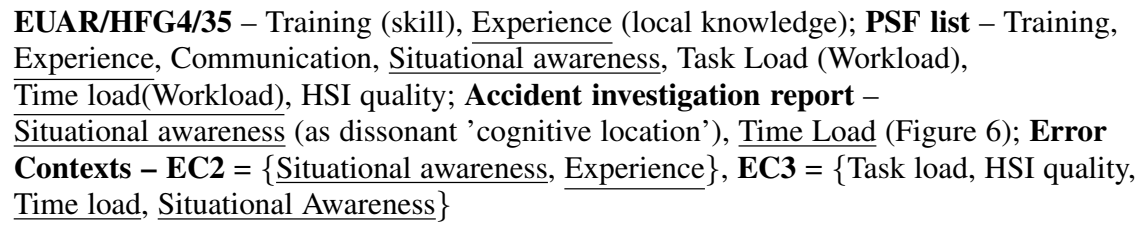 & $\begin{array}{l}H F T C_{H F E 3}=\{\text { Situational } \\
\text { Awareness, Time Load, } \\
\text { Experience }\}\end{array}$ \\
\hline
\end{tabular}


TABLE 10: Context description and questions for HFE2 sent to the experts

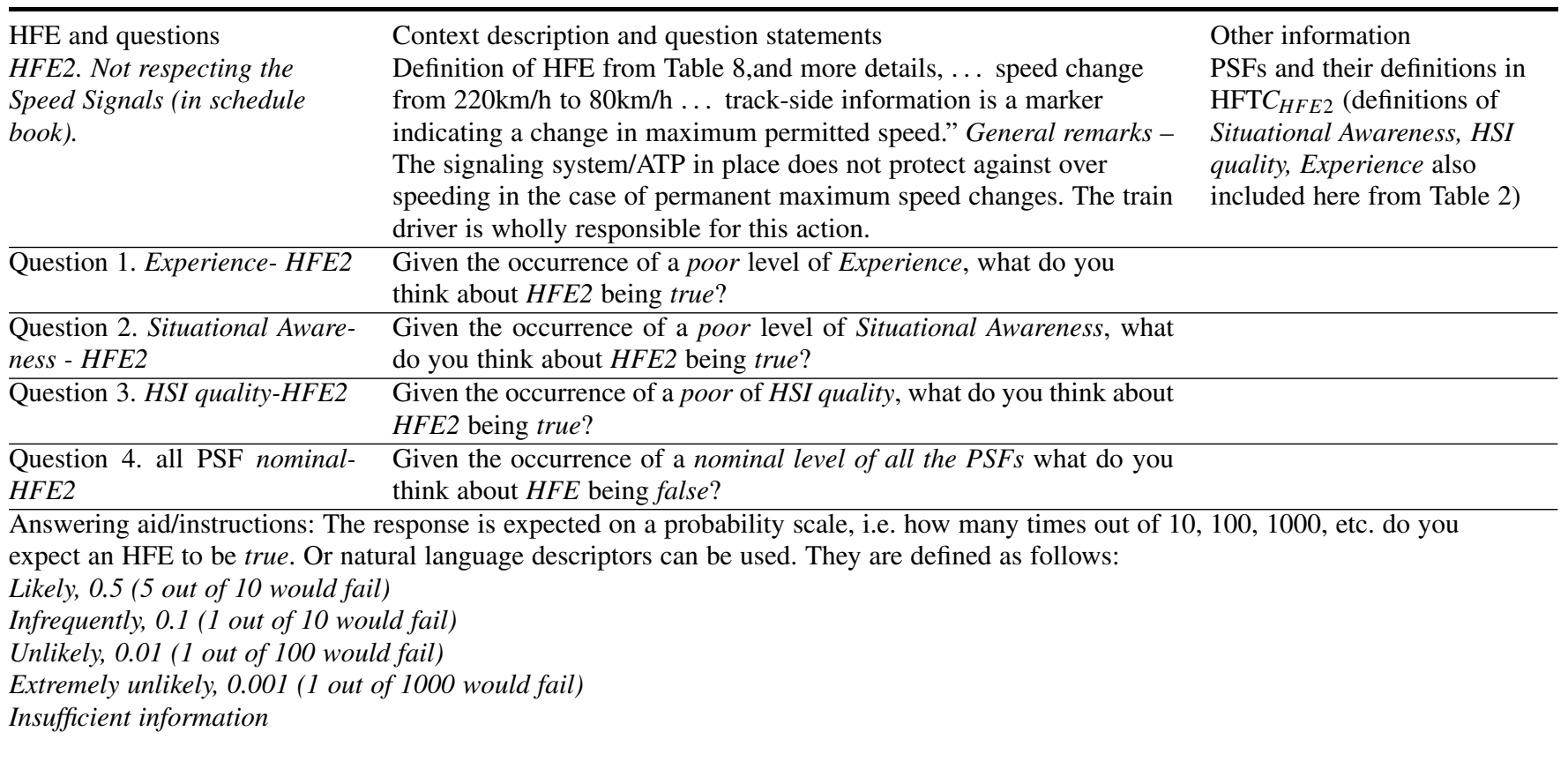

From a functional perspective, the act of braking and respecting a speed signal forms one single function. In the EUAR HF study the scope of human function (HFG4/35) entails both HFE2 and HFE3. Since present work is concerned with a detailed and precise analysis of the accident scenario; hence, they were quantified as separate HFEs. An appropriate granularity level in accordance with analysis objectives and thus, appropriate source of data must be selected. Current approach, takes such data from multiple sources to precisely identify the PSFs involved. Such, details might not be otherwise observed, if a single point of view is taken.

\subsection{Step 2. Quantitative part: Expert elicitation, data combination and transformation}

Three experts with different domain expertise were consulted. Their combined expertise covers human factors engineering, railway signaling, BFT, and safety and reliability aspects of the railway domain in general. Such a variety of domain knowledge is in-line with what is advised by other such expert-data based methods. Further, independent elicitations were carried out. The experts were sent the questions and related context detail. Table 10 shows the questions and descriptions for HFE2. Similar structure was followed for other HFEs identified in Step 1.

The responses received were a mix of numerical values and descriptors (numerical equivalents therefore were used). The numerical values thus obtained, for each question from the three experts $\mathrm{A}, \mathrm{B}$, and $\mathrm{C}$ are given in Figure 7. Subsequently, data from each expert for each question was combined using different combination rules (section 2.3.2). For weighted average (WA) combination a choice was made to give a higher weight to expert with experience in the railway industry (expert $\mathrm{C}$ ). Thus, the following normalized weighting factors were chosen: 0.2 for expert $\mathrm{A}$ and $\mathrm{B}$, and 0.6 for expert $\mathrm{C}$. The combined values for each question thus obtained is also given in Figure 7 .

Separate belief structures were generated (section 2.3.3 for each of the five combination methods. That is for each combination method used, different $b p a$ was generated for each HFE's VBS model. Expanding on the discussion of section 2.3.2, here we briefly comment on the different combination rules to demonstrate the difference. The Figure 7 shows in the form of the grouped bar plots for each question - the expert data (first three bars) and the data obtained after different combination rules (latter bars in the same group). The expert data for 


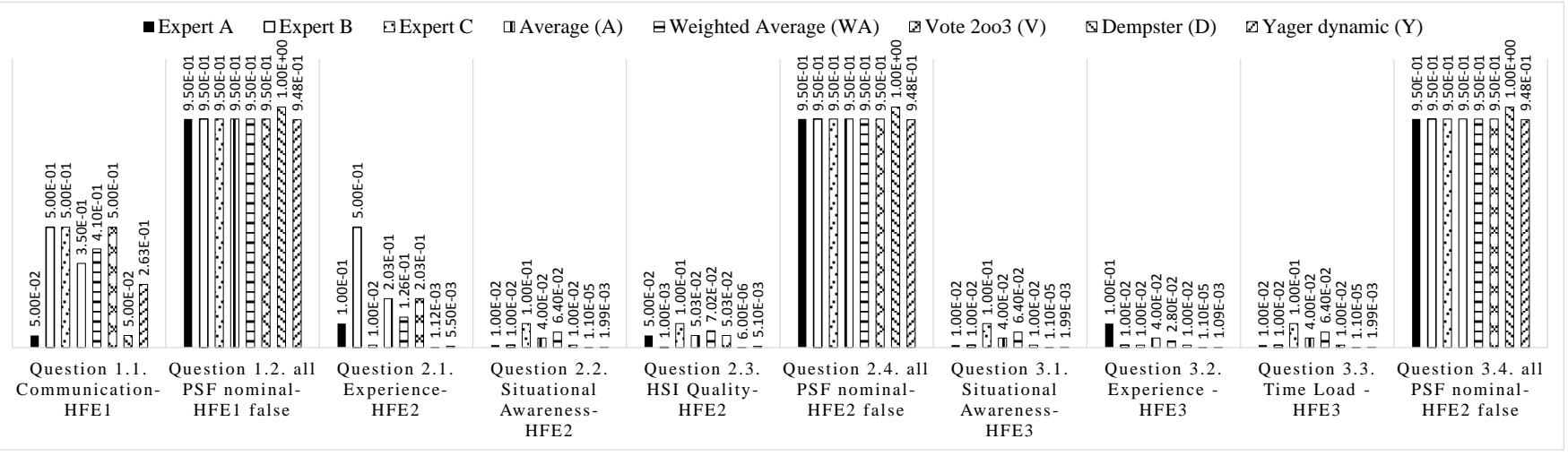

Fig. 7: Expert data (first three bars) and data obtained from the combination rules, for the case study.

TABLE 11: Direct belief structures (focal set and BPA) for the PSFs in the case study, identified similar to as in Table 6 from the R-PSF equivalent

\begin{tabular}{llrlr}
\hline $\begin{array}{l}\text { PSFs used in the case } \\
\text { study }\end{array}$ & $\begin{array}{l}\text { BPA for focal } \\
\left.m^{P S F i}(\{(\text { poor })\})\right)\end{array}$ & set & $\begin{array}{l}\text { BPA for focal } \\
m^{P S F i}(\{(\text { nominal })\})\end{array}$ & set \\
\hline Communication & 0.136 & 0.864 & \\
Experience & 0.0787 & 0.9213 & \\
Situational Awareness & 0.144 & 0.856 & \\
HSI Quality & 0.1885 & 0.8115 & \\
Time Load & 0.0173 & 0.9827 & \\
\hline
\end{tabular}

question 1.1. is commented here, similar comments can be made for other questions. It can be observed that expert $\mathrm{B}$ and $\mathrm{C}$ give the same probability value, whereas expert A gives a significantly lower probability value. This discussion is from the perspective of what the experts say (the probability values they give) and the combined data that is obtained.

- A weighted average is different than simple average since it enriches an average by taking into account the expertise of an expert (higher weight).

- A vote tends to account for essentially what majority of experts say, irrespective of their expertise (weight) or the difference between the values themselves.

- In Dempster's rule the difference between the values is interpreted as as conflict $(k)$. Subsequently, Dempster's rule manages this conflict by normalizing it. The higher the conflict, the more normalization is performed. There is a conflict in this case, thus, a value smaller than previous combination rules is obtained.

- Furthermore, non-idempotent nature of Dempster's and Yager's rule (Table 5])gives some interesting results for question 1.2., 2.4. and 3.4.

- Yager's rule computes the conflict similarly, but treats it as an uncertainty instead of normalizing it. Therefore, a value higher than Dempster's rule is obtained. It may be noted that the version of Yager's rule (dynamic) used here is quasi-associative (Table 5). That is the order in which the expert data is combined has an influence on the combination result. Thus we see that, for question 3.1. vs. 3.2. Yager gives different results (1.99E-03 vs. 1.09E-03).

When the conflict is low, (and all the experts are reliable) under situations of minimal or irrelevant conflict and all of the sources can be considered reliable, a Dempster combination might be justified.

Here the frames for HFE2 and HFE3 were considered same as the example HFE in section 2.3.4, that is $\Omega_{\mathrm{HFE}}=\{$ true, false $\}$, and for PSFs as $\Omega_{\mathrm{PSFi}}=\{$ nominal, poor $\}$. With the exception of HFE1, here, $\Omega_{\mathrm{Communication}}=$ $\{$ good,nominal,poor $\}$, to demonstrate the ability to work with multi-state PSFs. The level good was treated 

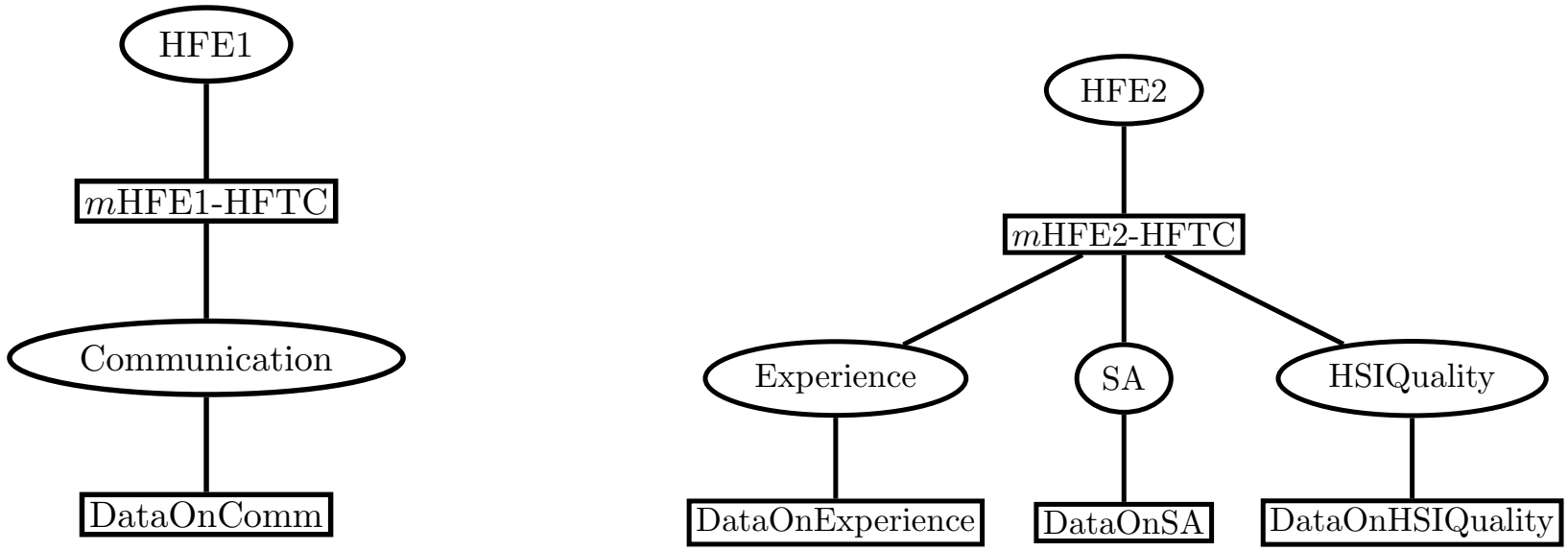

Fig. 8: VBS model of HFE1 and its HFTC. Fig. 9: VBS model of HFE2 and its HFTC.

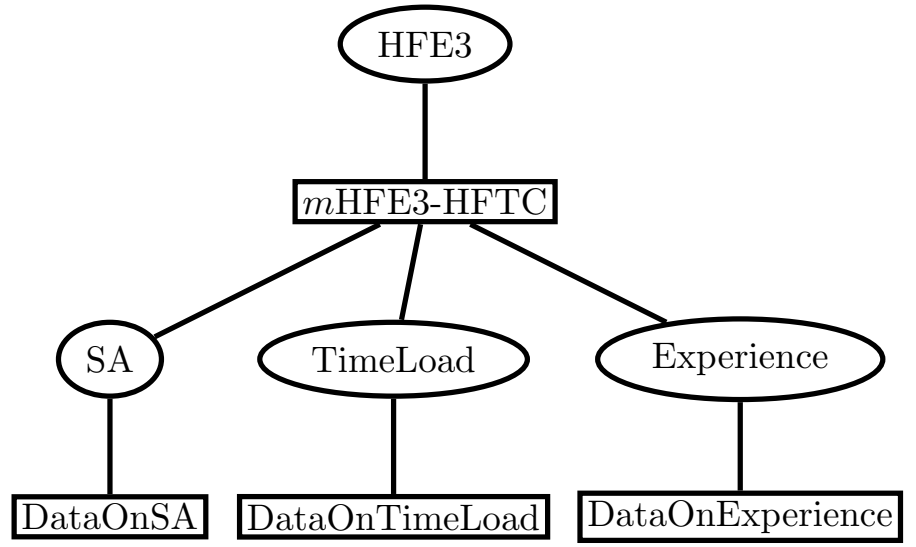

Fig. 10: VBS model of HFE3 and its HFTC.

similar to nominal as far as questions to the experts and quantification data (direct belief structure given in Table 11) are concerned. Configuration belief structures were defined and transformed the same way as other questions (following steps of section 2.3.3). For all the HFEs the modeling in VBS is thus complete. The implementation in VBS is given for HFE1 in Figure 8, HFE2 in Figure 9 and HFE3 in Figure 10. It can be noted that the human reliability models thus constructed are not specific to the case study. It can be used wherever similar HFEs and PSFs are identified. The quantification results and feedback/sensitivity analysis results are presented and discussed in the next step.

\subsection{Step 3. Quantification data and results}

Finally, in the last column of Table 11 the data for quantification, i.e. direct evidence, for respective direct belief structures are obtained from accident statistics data (explained in section 2.3.4). This data for quantification is assigned to all the PSFs of the case study (data on PSFs as seen in Figure 8,9 and 10 ).

Each of the combination rules used gives a different result. However, one combination rule can be chosen to obtain the necessary results i.e. an interval for each state of the variable of interest (an HFE). Nevertheless, 
TABLE 12: Top: Middle of the probability interval for the variable HFE1 value of interest (true) as obtained after combining data using different combination rules. Bottom: The pairwise distance metric $d_{J}$ from equation 2 between the BPAs obtained for HFE1.

\begin{tabular}{lllllll}
\hline Middle of interval & mAverage & mWeightedAverage & mVote & mDempsters & mYagers \\
\hline for HFE1(true) & 0.113 & & 0.117 & 0.124 & 0.071 & 0.108 \\
\hline & & & & & \\
\hline BPA obtained from & mAverage & mWeightedAverage & mVote & & mDempsters \\
combination rule & & & & - & - \\
\hline mWeightedAverage & 0.797 & 0.799 & - & - \\
mVote & 0.797 & 0.819 & 0.821 & - \\
mDempsters & 0.817 & 0.799 & 0.801 & 0.82178 \\
mYagers & 0.797 & & & & \\
\hline
\end{tabular}

towards PRELUDE's guide on the choice of combination methods (in addition to discussion in section 2.3.2) a brief discussion is presented here. This comparison is different than what was discussed in the previous section, where the combination of expert data and immediate results were discussed. Further, in this case the combination rules are compared at the level of end-results, which are BPAs obtained for the HFE. Only HFE1 are discussed here, similar metrics can be computed for other HFEs.

Table 12 gives the middle of the interval for the variable of interest HFE1(true) as described in section 2.1.3. In this case the true value of HFE is chosen. It can be remarked that the middle of the interval for all the combination rules are similar except for Dempster's rule. This is because of the way in which it manages conflict is different than Yager's rule, and others which do not manage conflict explicitly (see section 2.3.2).

The distance metric $d_{J}$ can take a maximum value of 1 [77]. Thus we can observe in Table 12 that all of the BPAs have high distance values between them, that is they are dissimilar pairwise. However, relatively speaking each BPA is at equal (maximum distances between two BPAs 0.821 and minimum 0.797) distances from each other. In this case, the distances are not significantly informative, they do not provide sufficient information to single out particular rules or a pair thereof which is dissimilar/similar to the others. Nevertheless, such a metric can be useful when it is difficult to make a choice of combination rule only based on the underlying hypothesis.

The quantification results are represented in the form of upper and lower probability bound. The value of variable of interest is an HFE's true state. i.e. HFE(true). The quantification results are given in Figure 11. The $x$ axis marks the combination method used and the $y$ axis is the probability value on a $\log _{10}$ scale, with an axis maximum of 0.2. The line-plot in $y$ axis presents the log of lower and upper probability bound of $H F E($ true $)$. The lower bounds for first three combination rules are of the order of $10^{-3}$, however for latter two (BFT-based) it was $10^{-6}$. Generally this is also the case with human error probabilities in other HRA methods. Nevertheless, for all intents and purposes, the values obtained are indicative than representative.

The results of HFE1(true) have higher upper bounds due to the higher values obtained (an average of 0.35) obtained from the experts. For both HFE2(true) and HFE3(true) in terms of interval size and lower bounds, a clear distinction must be made between BFT-based (Dempster's and Yager's), and other combination rules. This is due to the latter with implicit and the former with explicit handling of conflict. Weighted average implicitly removes conflict by weighing values obtained from one expert (Expert $\mathrm{C}$, for present case, Figure 7) more than others. Nevertheless, the intervals are not completely disconnected since conflict is relatively less; if it was higher significant changes could have been observed. Therefore, simpler combination methods are as effective when conflict is low or ignorable. In the case where (HFE2 and HFE1) lower bound for Dempster's rule is smaller than Yager's; this is due to the presence of high conflict in elicited data (ref. Figure 7). In Yager's hypothesis, the conflict amongst expert's values is distributed as uncertainty, instead of normalizing the lower bound. Thus, in both the cases of HFE2(true) and HFE3(true), Yager's rule gives a larger interval size as compared to Dempster's results. That is, even though it is an accurate/formal representation of uncertainty, in some cases it might make decision-making difficult. 

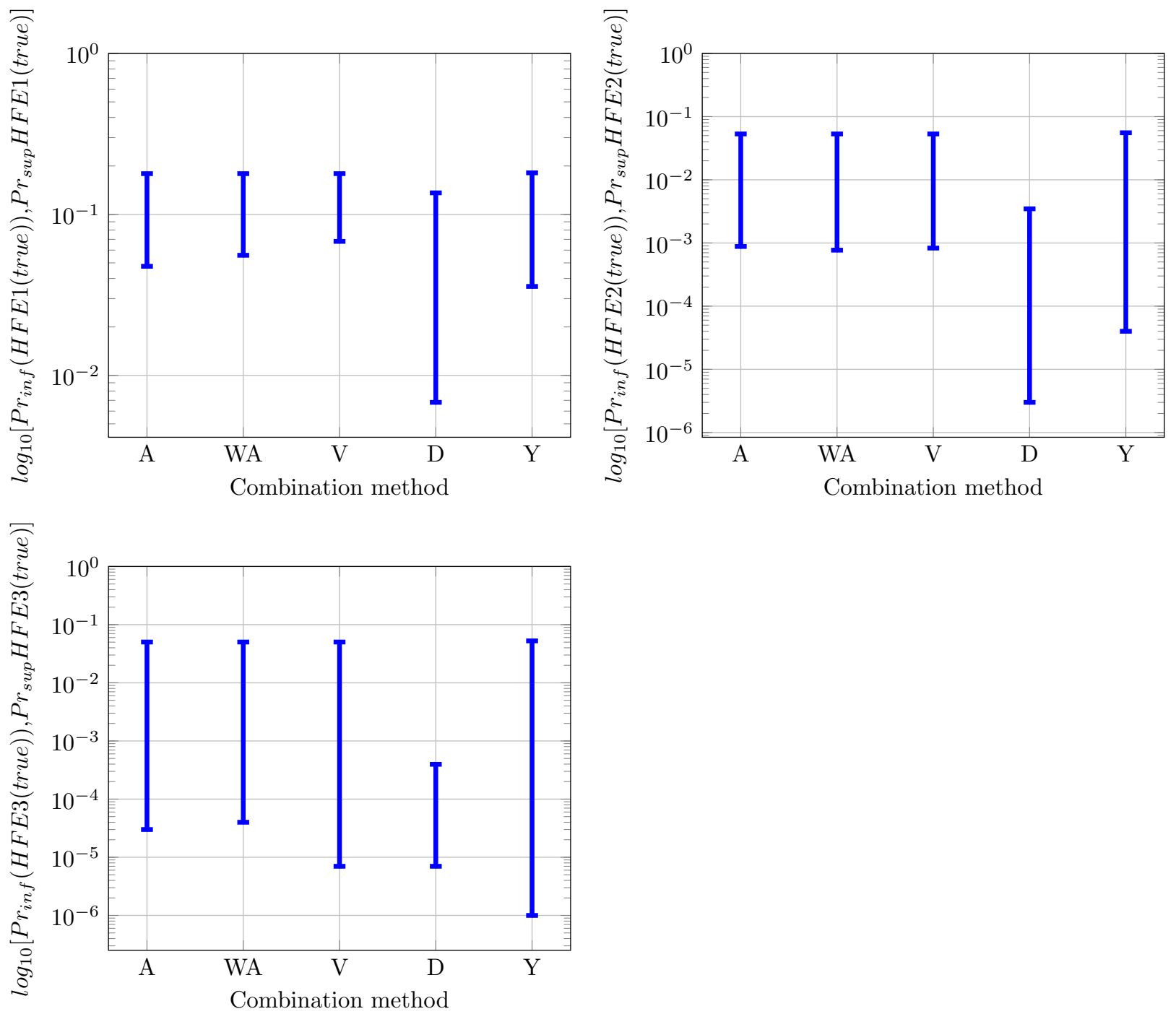

Fig. 11: Lower and upper bounds for an HFE's true state from VBS models obtained after combining expert elicitation using average (A), weighted average (WA), vote/independent consensus (V), Dempster's (D) and Yager's (Y) combination rules.

Towards keeping this choice open, PRELUDE methodology remains adaptable and only needs conditional quantitative data on a PSF-HFE pair. The transformation (section 2.3.3 manages the rest to construct the VBS model. This also ensures that explicit considerations of epistemic uncertainty (in the human error relational model) are uniform irrespective of the combination rule used. Subsequently, for HFE2 and HFE3 a sensitivity analysis (following the steps in section 2.3.4.1) is undertaken. HFE1 is not analyzed further because it contains only one PSF.

It can be noted that for each expert data combination method, different VBS models (configuration belief structures) are generated, for space constrains it is not possible to discuss all of them here. Hence, only average combination rule is selected for both HFE and only the poor level of a PSF is considered. Figure 12 shows the obtained sensitivity analysis results. They are presented in the form of a relative percentage value, interpreted as a relative contribution towards causing an HFE to be true. It can be seen that, HSI quality for HFE2 and Situational Awareness for $H F E 3$, have the highest relative contribution. Hence, priority focus should be on improving aspects 

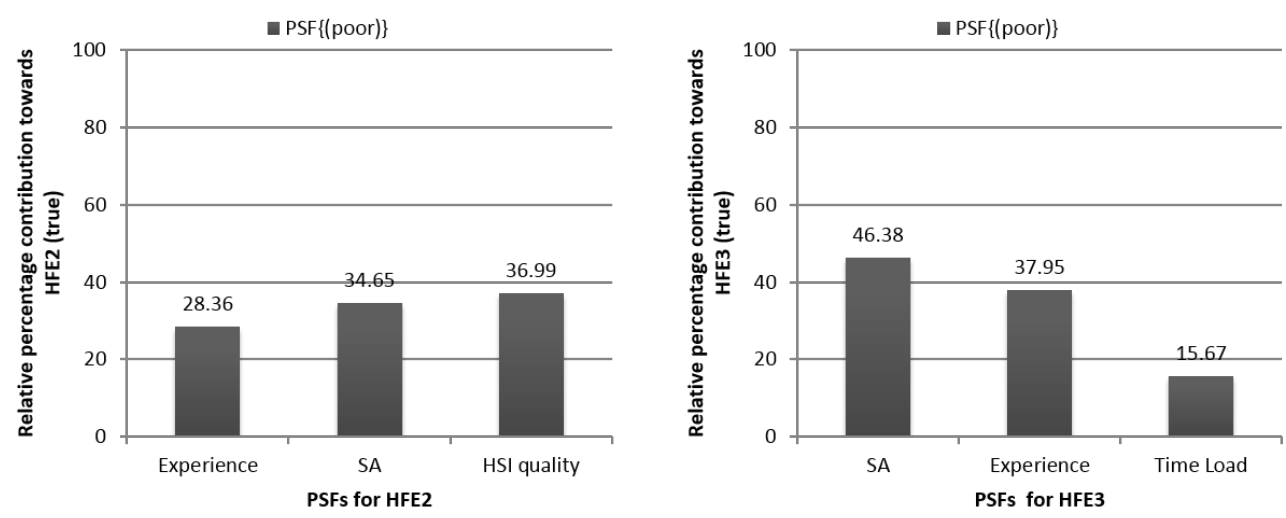

Fig. 12: Sensitivity analysis results of PSFs for the context of $H F E 2$ and HFE3.

\section{of HSI quality and Situational Awareness.}

The results effectively are also indicative of the reality (the accident investigation report) and expectations of experts. HFE2 can be classified as a "checking error" [86]; for a checking error the quality or source of information (i.e. HSI quality) can be judged to be relatively critical than other factors. This corresponds to the results seen in Figure 12. Thus, it can be remarked that the model gives results corresponding to conclusions from the human error mechanisms. Furthermore, these results are also reaffirmed in the accident analysis report. The investigators concluded that the availability of right information at the right time was indeed one of the issues. Subsequently, a change of the placement of track-side speed markers was ordered. In particular, special considerations (more trackside markers, gradual speed changes, etc.) are made for places where a high change in ceiling speed ( $200 \mathrm{~km} / \mathrm{h}$ to $80 \mathrm{~km} / \mathrm{h}$, blue line in Figure 6 is required. For HFE3, PRELUDE's analysis concludes that Situational Awareness has the highest relative contribution. This also, corresponds to the concluding statement of the report that absence of attention was one of the main causes of the accident. Thus, the proposed quantitative model presents results coherent with expert and domain knowledge.

\section{Conclusions AND PERSPeCtives}

PRELUDE methodology in its current state is developed for and applied to a case study for the railway domain. It may very well (with moderate efforts) be applied to other domains. A completely generic HRA model is rare (table 1), and such models are often inspired by the notions of previous domain-specific HRA models.

It entails an expert system built using concepts from BFT in VBS which formally defines the casual elements of a human reliability model. Decision-making capabilities are provided by using probability intervals and sensitivity analysis to establish a priority ranking amongst the PSFs for a given safety critical situation. PRELUDE is centered on Performance Shaping Factors specific for railway needs. PSFs and human factor studies are employed for an adequate representation of human factors and operational safety concerns. It was also demonstrated as how to employ human factor study towards HRA objectives. The expert system's graphical representation as a VBS allows for an easy representation of variables and their relationships, and, thus simplifies the usage by analysts and non-experts in the mathematical framework.

A formal combination and transformation proposal is used to build the elements of quantitative human reliability model from expert data. This approach to formally model human failure events as a function of the PSFs in evidential networks (VBS) offers a novel perspective for human reliability quantification. The expert data can be replaced by an empirical source, with the condition that it is in the form of a conditional probability, at the very least HFE-PSF (the questions currently asked of the experts) or HFE-multiple PSFs. Conditional in terms of 
the variable HFE and PSF, the states thereof poor or nominal, true or false, are not a limitation to BFT framework. Thus, if the empirical data can be formulated as a (conditional) belief structure, it can be integrated into the VBS model currently proposed.

Although, when combining evidences (direct and configuration belief structures) the resulting frame is the product of the respective variables' frames, there the size of the product space may create a computational bottleneck. That is to say, more granular variables with larger frames (i.e. multiple states of PSFs and HFEs) are difficult to combine, compared to e.g. multiple variables (multiple PSFs and HFEs) with less number of states (smaller frames). Some rules for combination of expert elicited data are also contrasted. A relatively straightforward middle of the interval comparison, and a standardized distance metric are used. These metrics, as used in the context of present work allow comparing the results obtained by using different combination rules in a quantitative manner. It allows the experts to identify the appropriate choice of combination rule to use or justify the choice.

If particularly conflicting expert opinions are considered, it is observed that some methods result in larger intervals than others. Thus, the choice thereof is left to the analyst; nevertheless, the usage of such methods needs further investigation. Sensitivity analysis results were used to establish a priority rank towards improvements in PSFs needed for effective gains in human reliability. Although it is more interesting if multiple PSFs and multiple HFEs are modeled in the same EN, this might lead to non-evident sensitivity analysis results. The proposition's implementation on a retrospective analysis of a real-world railway accident demonstrated the usage of the methodology. Once the VBSs models are built the implementation is relatively straightforward, and interval provides accurate representation of uncertainty in data (if any) and easy decision making. The results obtained correspond to theoretical and expert expectations. Thus, VBS offers an adequate framework in its utility towards newer generation of human reliability methods.

System level, risk-based inferences, and taking contribution of positive aspects of PSFs and human actions need to be appended to PRELUDE towards a robust methodology. This is aimed at further simplifying the decisionmaking capabilities, and performing a holistic analysis of a human error. Feedback or remarks of the experts on the structure of questionnaire can also be obtained to improve the elicitation process, mainly to ensure that the analyst and experts have the same understanding towards assuring the accuracy of obtained data. Validation using simulator data and sensitivity analysis can be employed towards immediate verification objectives. Further, usage of empirical data from operational simulators to reinforce expert knowledge and to validate the methodology needs to be explored further.

\section{ACKNOWLEDGMENT}

This work was carried out and funded in the framework of the Labex MS2T. It was supported by the French Government, through the program "Investments for the future" managed by the National Agency for Research (Reference: ANR-11-IDEX-0004-02). It was also supported by the National Agency for Research, through the RECIF project (Reference: ANR-13-JS03-0007). The authors would also like to thank Dr. Prakash P. Shenoy for Belief Functions Machine. We would like to thank the associate editor, and all the anonymous reviewers for their time, valuable remarks, and comments.

\section{REFERENCES}

[1] US Nuclear Regulatory Commission Regulation, "Reactor Safety Study: An Assessment of Accident Risks in U.S. Commercial Nuclear Power Plants [NUREG-75/014 (WASH-1400)]," US Nuclear Regulatory Commission Regulation, Tech. Rep., 1975. [Online]. Available: http://www.nrc.gov/reading-rm/doc-collections/nuregs/staff/sr75-014/

[2] J. b. Zamanali, "Probabilistic-risk-assessment applications in the nuclear-power industry," IEEE Transactions on Reliability, vol. 47, no. 3 SPEC. ISS. 2, pp. SP-361-364-SP, 1998. [Online]. Available: http://www.scopus.com/inward/record.url?eid=2-s2. 0-0032156640 $\{\&\}$ partnerID $=40\{\&\} \mathrm{md5}=$ fad88ba17770b6e65403290820bafe94

[3] B. Kirwan and H. Gibson, "CARA: A Human Reliability Assessment Tool for Air Traffic Safety Management - Technical Basis and Preliminary Architecture," The Safety of Systems, pp. 197-214, 2007. [Online]. Available: http://link.springer.com/10.1007/978-1-84628-806-7\{_\}13

[4] A. J. Spurgin, Human Reliability Assessment Theory and Practice. CRC Press, 2009. 
[5] R. L. Boring, "Fifty Years of THERP and Human Reliability Analysis," in Proceedings of the Probabilistic Safety Assessment and Management and European Safety and Reliability Conference (PSAM 11 \& ESREL 2012). 2012, 2012, pp. 3523-3532.

[6] K. Lee, F. Tillman, and J. Higgins, "A literature survey of the human reliability component in a man-machine system," IEEE Transactions on Reliability, vol. 37, no. 1, pp. 24-34, apr 1988. [Online]. Available: http://ieeexplore.ieee.org/document/3708/

[7] F. Vanderhaegen, "Cooperation and learning to increase the autonomy of ADAS," Cognition, Technology \& Work, vol. 14, no. 1, pp. 61-69, sep 2011. [Online]. Available: http://link.springer.com/10.1007/s10111-011-0196-1]

[8] F. Vanderhaegen and S. Zieba, "Reinforced learning systems based on merged and cumulative knowledge to predict human actions," Information Sciences, vol. 276, pp. 146-159, aug 2014. [Online]. Available: http://linkinghub.elsevier.com/retrieve/pii/] S0020025514001571

[9] F. Vanderhaegen, D. Jouglet, and S. Piechowiak, "Human-Reliability Analysis of Cooperative Redundancy to Support Diagnosis," IEEE Transactions on Reliability, vol. 53, no. 4, pp. 458-464, dec 2004. [Online]. Available: http: //ieeexplore.ieee.org/lpdocs/epic03/wrapper.htm?arnumber=1360104

[10] F. Vanderhaegen and P. Caulier, "A multi-viewpoint system to support abductive reasoning," Information Sciences, vol. 181, no. 24, pp. 5349-5363, 2011.

[11] R. L. Boring, C. D. Griffith, and J. C. Joe, "The measure of human error: Direct and indirect performance shaping factors," in IEEE Conference on Human Factors and Power Plants, 2007, pp. 170-176.

[12] H. S. Blackman, D. I. Gertman, and R. L. Boring, "Human Error Quantification Using Performance Shaping Factors in the SPAR-H Method," in Proceedings of the Human Factors and Ergonomics Society Annual Meeting, vol. 52, no. 21, sep 2008, pp. 1733-1737. [Online]. Available: http://pro.sagepub.com/lookup/doi/10.1177/154193120805202109

[13] R. L. Boring, "How Many Performance Shaping Factors are Necessary for Human Reliability Analysis ?" in Proceedings of the 10th International Probabilistic Safety Assessment \& Management Conference (PSAM10), 2010, pp. 1479-1487.

[14] M. Kyriakidis, A. Majumdar, G. Grote, and W. Y. Ochieng, "Development and Assessment of Taxonomy for Performance-Shaping Factors for Railway Operations," SSRN Electronic Journal, vol. 2289, no. -1, pp. 145-153, 2011. [Online]. Available: http://www.scopus.com/inward/record.url?eid=2-s2.0-84869787021 \&\&\}partnerID=tZOtx3y1http://www.ssrn.com/abstract=2506591

[15] M. Kyriakidis, A. Majumdar, and W. Y. Ochieng, "Data based framework to identify the most significant performance shaping factors in railway operations," Safety Science, vol. 78, pp. 60-76, 2015. [Online]. Available: http://dx.doi.org/10.1016/j.ssci.2015.04.010

[16] P. Le Bot, "Human reliability data, human error and accident models-illustration through the Three Mile Island accident analysis," Reliability Engineering \& System Safety, vol. 83, no. 2, pp. 153-167, 2004.

[17] A. Wang, Y. Luo, G. Tu, and P. Liu, "Quantitative evaluation of human-reliability based on fuzzy-clonal selection," IEEE Transactions on Reliability, vol. 60, no. 3, pp. 517-527, 2011.

[18] B. Kirwan, H. Gibson, R. Kennedy, J. Edmunds, G. Cooksley, and I. Umbers, "Nuclear Action Reliability Assessment (NARA): A Data-Based HRA Tool," in Probabilistic Safety Assessment and Management. London: Springer London, 2004, pp. 1206-1211. [Online]. Available: http://link.springer.com/10.1007/978-0-85729-410-4\{_\}195

[19] K. M. Groth and A. Mosleh, "Deriving causal Bayesian networks from human reliability analysis data: A methodology and example model," Proceedings of the Institution of Mechanical Engineers, Part O: Journal of Risk and Reliability, vol. 226, no. 4, pp. 361-379, aug 2012. [Online]. Available: http://pio.sagepub.com/lookup/doi/10.1177/1748006X11428107

[20] J. Forester, D. Bley, S. Cooper, E. Lois, N. Siu, A. Kolaczkowski, and J. Wreathall, "Expert elicitation approach for performing ATHEANA quantification," Reliability Engineering \& System Safety, vol. 83, no. 2, pp. 207-220, feb 2004. [Online]. Available: http://linkinghub.elsevier.com/retrieve/pii/S0951832003002072

[21] J. Forester, A. Kolaczkowski, S. Cooper, D. Bley, and E. Lois, “ATHEANA User's Guide Final Report, NUREG-1880,” U.S. Nuclear Regulatory Commission, Tech. Rep., 2007. [Online]. Available: http://www.nrc.gov/reading-rm/doc-collections/nuregs/staff/sr1880

[22] S. E. Cooper, A. M. Ramey-Smith, J. Wreathall, G. W. Parry, D. C. Bley, W. J. Luckas, J. H. Taylor, and M. T. Barriere, "A Technique for Human Error Analysis (ATHEANA)," U.S. Nuclear Regulatory Commission, Brookhaven National Laboratory, Upton, Tech. Rep., 1996.

[23] B. Reer, "Review of advances in human reliability analysis of errors of commission-Part 2: EOC quantification," Reliability Engineering and System Safety, vol. 93, no. 8, pp. 1105-1122, 2008.

[24] X. Su, S. Mahadevan, P. Xu, and Y. Deng, "Dependence Assessment in Human Reliability Analysis Using Evidence Theory and AHP," Risk Analysis, vol. 35, no. 7, pp. 1296-1316, 2015.

[25] M. C. Kim, P. H. Seong, and E. Hollnagel, "A probabilistic approach for determining the control mode in CREAM," Reliability Engineering and System Safety, vol. 91, no. 2, pp. 191-199, 2006.

[26] A. M. Whaley, D. L. Kelly, R. L. Boring, and W. J. Galyean, "SPAR-H Step-by-Step Guidance," Idaho National Laboratory, Tech. Rep. May, 2011.

[27] H. S. Blackman and D. I. Gertman, Human reliability and safety analysis data handbook. John Wiley \& Sons, 1994.

[28] T. Aven, "Interpretations of alternative uncertainty representations in a reliability and risk analysis context," Reliability Engineering and System Safety, vol. 96, no. 3, pp. 353-360, 2011. [Online]. Available: http://dx.doi.org/10.1016/j.ress.2010.11.004

[29] G. W. Parry, "The characterization of uncertainty in probabilistic risk assessments of complex systems," Reliability Engineering and System Safety, vol. 54, no. 2-3, pp. 119-126, 1996.

[30] G. Apostolakis, "The concept of probability in safety assessments of technological systems," Science, vol. 250, no. 4986, pp. 1359-1364, 1990. [Online]. Available: http://science.sciencemag.org/content/250/4986/1359

[31] T. Aven and E. Zio, "Some considerations on the treatment of uncertainties in risk assessment for practical decision making," Reliability Engineering and System Safety, vol. 96, no. 1, pp. 64-74, 2011. 
[32] R. G. Almond, Graphical Belief Modeling, 1st ed. Boca Raton, FL, USA: CRC Press, Inc., 1995.

[33] P. Baraldi, L. Podofillini, L. Mkrtchyan, E. Zio, and V. N. Dang, "Comparing the treatment of uncertainty in Bayesian networks and fuzzy expert systems used for a human reliability analysis application," Reliability Engineering \& System Safety, vol. 138, pp. 176-193, 2015. [Online]. Available: http://linkinghub.elsevier.com/retrieve/pii/S0951832015000265

[34] H. Langseth and L. Portinale, "Bayesian networks in reliability," Reliability Engineering \& System Safety, vol. 92, no. 1, pp. 92-108, 2007. [Online]. Available: http://linkinghub.elsevier.com/retrieve/pii/S0951832005001985

[35] M. R. Martins and M. C. Maturana, "Application of Bayesian Belief networks to the human reliability analysis of an oil tanker operation focusing on collision accidents," Reliability Engineering and System Safety, vol. 110, pp. 89-109, 2013. [Online]. Available: http://dx.doi.org/10.1016/j.ress.2012.09.008

[36] L. Mkrtchyan, L. Podofillini, and V. Dang, "Bayesian belief networks for human reliability analysis: A review of applications and gaps," Reliability Engineering \& System Safety, vol. 139, pp. 1-16, jul 2015. [Online]. Available: http://www.sciencedirect.com/science/article/pii/S0951832015000514http://linkinghub.elsevier.com/retrieve/pii/S0951832015000514

[37] M. De Ambroggi and P. Trucco, "Modelling and assessment of dependent performance shaping factors through Analytic Network Process," Reliability Engineering \& System Safety, vol. 96, no. 7, pp. 849-860, jul 2011. [Online]. Available: http://dx.doi.org/10.1016/j.ress.2011.03.004http://linkinghub.elsevier.com/retrieve/pii/S0951832011000329

[38] L. Podofillini and V. Dang, "A Bayesian approach to treat expert-elicited probabilities in human reliability analysis model construction," Reliability Engineering \& System Safety, vol. 117, pp. 52-64, 2013. [Online]. Available: http://www.sciencedirect.com/science/article/pii/S0951832013000914

[39] F. Ouchi and W. Bank, A Literature Review on the Use of Expert Opinion in Probabilistic Risk Analysis. World Bank Publications, jan 2004, vol. 3201, no. February. [Online]. Available: http://econpapers.repec.org/RePEc:wbk:wbrwps:3201http://elibrary.worldbank.org/rpsv/cgi-bin/wpapers?ID=1012480 $\{\&\}$ br= $\mathrm{a}\{\&\}$ mode $=$ browse $\{\&\} \mathrm{bsd}=1\{\&\}$ ini=wb $\{\&\} \mathrm{ss}=$ title $\{\%\} 5 \mathrm{Ea}^{*}\{\&\} \mathrm{bb}=$ title $\{\&\} \mathrm{sd}=1\{\&\} \mathrm{th}=73\{\&\} \mathrm{hn}=1\{\&\}$ browseview $=3201$

[40] A. W. Evans, "Fatal train accidents on Europe's railways: 1980-2009." Accident; analysis and prevention, vol. 43, no. 1, pp. 391-401, jan 2011. [Online]. Available: http://www.ncbi.nlm.nih.gov/pubmed/21094337

[41] B. S. Dhillon, Human Reliability and Error in Transportation Systems, ser. Springer Series in Reliability Engineering. London: Springer London, 2007. [Online]. Available: http://link.springer.com/10.1007/978-1-84628-812-8

[42] M. Kyriakidis, K. T. Pak, and A. Majumdar, "Railway Accidents due to Human Error A historic analysis of the UK railways ( 1945-2012 )," Transportation Research Record: Journal of the Transportation Research Board, pp. 1-16, 2015. [Online]. Available: http://ssrn.com/abstract=2572898

[43] T. Lindner, B. Milius, D. Schwencke, and K. Lemmer, "Influential factors on human performance in railways and their interrelations," in Safety, Reliability and Risk Analysis. CRC Press, sep 2013, no. SEPTEMBER, pp. 435-442. [Online]. Available: http://www.crenetbase.com/doi/abs/10.1201/b15938-69

[44] D. Schwencke, T. Lindner, B. Milius, M. Arenius, O. Sträter, and K. Lemmer, "A new method for human reliability assessment in railway transport," in Annual European Safety and Reliability Conference ESREL 2012, vol. 8, no. 2, 2012, pp. 6139-6147.

[45] F. Feldmann, M. Hammerl, and S. Schwartz, "Questioning human error probabilities in railways," in 3rd IET International Conference on System Safety 2008. IEE, 2008, pp. 4B2-4B2. [Online]. Available: http://digital-library.theiet.org/content/conferences/10.1049/ cp $\left\{\_\right\} 20080728$

[46] S. Rangra, M. Sallak, W. Schön, and F. Vanderhaegen, "On the study of human reliability in transportation systems of systems," in 2015 10th System of Systems Engineering Conference (SoSE). San Antonio, TX, USA: IEEE, may 2015, pp. 208-213. [Online]. Available: http://ieeexplore.ieee.org/lpdocs/epic03/wrapper.htm?arnumber=7151980

[47] M. Hammerl and F. Vanderhaegen, "Human factors in the railway system safety analysis process," in 3rd International Rail Human Factors Conference, vol. 1, 2009, pp. 1-9.

[48] H. W. Gibson, A. Mills, S. Smith, and B. K. Kirwan, "Railway Action Reliability Assessment A Railway - Specific Approach to Human Error Quantification," Rail Human Factors Supporting Reliability, Safety and Cost Reduction, pp. 671-676, 2013.

[49] J. Williams, "HEART - A proposed method for achieving high reliability in process operation by means of human factors engineering technology," in Proceedings of a Symposium on the Achievement of Reliability in Operating Plant, Safety and Reliability Society (SaRS), NEC, Birmingham, 1985.

[50] E. Castillo, Z. Grande, and A. Calviño, "Bayesian Networks-Based Probabilistic Safety Analysis for Railway Lines," Computer-Aided Civil and Infrastructure Engineering, vol. 31, no. 9, pp. 681-700, 2016. [Online]. Available: http://doi.wiley.com/10.1111/mice.12195

[51] M. Kyriakidis, A. Majumdar, G. Grote, and W. Y. Ochieng, "Development and Assessment of Taxonomy for Performance-Shaping Factors for Railway Operations," Transportation Research Record: Journal of the Transportation Research Board, vol. 2289, pp. 145-153, 2012. [Online]. Available: http://www.scopus.com/inward/record.url?eid=2-s2.0-84869787021\{\&\}partnerID=tZOtx3y1

[52] M. Kyriakidis, "Developing a human performance railway operational index to enhance safety of railway operations," Imperial College London, no. October 2013, 2013. [Online]. Available: http://hdl.handle.net/10044/1/21760

[53] A. O. Mowitz and L. Kecklund, "Study on the Assessment and the Acceptance of Risks Related to Human Interactions within the European Railways - Final report - ERA/2011/SAF/OP/02,” European Railway Agency, Tech. Rep. January, 2013. [Online]. Available: http://www.era.europa.eu/Document-Register/Pages/Human-RAC-study.aspx

[54] European Railway Agency, "Recommendation of the European Railway Agency on the amendment of the Commission implementing Regulation (EU) No 402/2013 on the common safety method for risk evaluation and 口 assessment," European Railway Agency, Tech. Rep., 2015. [Online]. Available: http://www.era.europa.eu/Document-Register/ 
Pages/Recommendation-of-the-European-Railway-Agency-on-the-amendment-of-the-Commission-implementing-Regulation-(EU) -No-4022013-on.aspx

[55] A. Swain and H. Guttmann, "Handbook of human-reliability analysis with emphasis on nuclear power plant applications. Final report," Sandia National Labs., Albuquerque, NM (USA), Tech. Rep., 1983.

[56] D. E. Embrey, P. Humphreys, E. A. Rosa, B. Kirwan, and K. Rea, "SLIM-MAUD: An Approach to Assessing Human Error Probabilities Using Structured Expert Judgment, Volume 2: Detailed Analysis of the Technical Issues NUREG/CR-3518," Brookhaven National Laboratory, Brookhaven National Laboratory, Upton, Tech. Rep., 1984.

[57] M. Kyriakidis, A. Majumdar, and W. Y. Ochieng, "A human performance operational railway index to estimate operator's error probability," Advances in Human Aspects of Road and Rail Transportation, pp. 832-841, 2012.

[58] E. Hollnagel, Cognitive reliability and error analysis method (CREAM). Elsevier, 1998.

[59] M. Marseguerra, E. Zio, and M. Librizzi, "Human reliability analysis by fuzzy "CREAM"," Risk Analysis, vol. 27, no. 1, pp. 137-154, 2007.

[60] C. Bieder, P. Le-Bot, E. Desmares, J. L. Bonnet, and F. Cara, “MERMOS: EDF's new advanced HRA method,” p. $1998,1998$.

[61] M. J. Barnes, D. Bley, and S. Cooper, "Technical basis and implementation guidelines for a technique for human event analysis (ATHEANA),” NUREG-1624, Rev, 2000. [Online]. Available: http://scholar.google.com/scholar?hl=en $\{\&\}$ btnG=Search $\{\&\} \mathrm{q}=\mathrm{intitle:}$ Technical+Basis+and+Implementation+Guidelines+for+A+Technique+for+Human+Event+Analysis $\{\#\} 0$

[62] D. Gertman, H. Blackman, J. Marble, J. Byers, and C. Smith, "The SPAR-H human reliability analysis method," Idaho National Laboratory U.S., Tech. Rep., 2005. [Online]. Available: http://www.nrc.gov/reading-rm/doc-collections/nuregs/contract/cr6883/

[63] K. M. Groth and L. P. Swiler, "Bridging the gap between HRA research and HRA practice: A Bayesian network version of SPAR-H," Reliability Engineering and System Safety, vol. 115, pp. 33-42, 2013. [Online]. Available: http://dx.doi.org/10.1016/j.ress.2013.02.015

[64] T. Chandler, J. Chang, A. Mosleb, M. J., R. Boring, and D. Gertman, "Human Reliability Analysis Methods Selection Guidance for NASA," National Aeronautics and Space Administration, no. July, p. 175, 2006. [Online]. Available: http://www.hq.nasa.gov/office/codeq/rm/docs/HRA\{_\}Report.pdf

[65] A. P. Dempster, "Upper and lower probabilities induced by a multivalued mapping," The Annals of Mathematical Statistics, vol. 38, no. 2, pp. 325-339, 1967. [Online]. Available: http://www.jstor.org/stable/2239146

[66] G. Shafer, A mathematical theory of evidence. Princeton: Princeton university press, 1976.

[67] P. Smets, "Practical Uses of Belief Functions," Uncertainty in Artificial Intelligence 15. UAI99, pp. 612-621, 1999.

[68] M. Sallak, W. Schön, and F. Aguirre, "Extended component importance measures considering aleatory and epistemic uncertainties," IEEE Transactions on Reliability, vol. 62, no. 1, pp. 49-65, 2013.

[69] S. Qiu, R. Sacile, M. Sallak, and W. Schön, "On the application of Valuation-Based Systems in the assessment of the probability bounds of Hazardous Material transportation accidents occurrence,” Safety Science, vol. 72, pp. 83-96, feb 2015. [Online]. Available: http://dx.doi.org/10.1016/j.ssci.2014.08.006http://linkinghub.elsevier.com/retrieve/pii/S0925753514001878

[70] P. P. Shenoy, “A valuation-based language for expert systems," International Journal of Approximate Reasoning, vol. 3, no. 5, pp. 383-411, sep 1989. [Online]. Available: http://linkinghub.elsevier.com/retrieve/pii/0888613X89900091

[71] — - "Valuation-Based Systems for Bayesian Decision Analysis," Operations Research, vol. 40, no. 3, pp. 463-484, jun 1992. [Online]. Available: http://pubsonline.informs.org/doi/abs/10.1287/opre.40.3.463

[72] _ - "Using dempster-shafer's belief-function theory in expert systems," Advances in the Dempster-Shafer Theory of Evidence, pp. 395-414, 1994.

[73] H. Xu and P. Smets, "Reasoning in evidential networks with conditional belief functions," International Journal of Approximate Reasoning, vol. 14, no. 2-3, pp. 155-185, feb 1996. [Online]. Available: http://www.sciencedirect.com/science/article/pii/ 0888613X96001132http://linkinghub.elsevier.com/retrieve/pii/0888613X96001132

[74] K. Sentz and S. Ferson, "Combination of Evidence in Dempster- Shafer Theory," Sandia National Laboratories, Tech. Rep. Tech. Rep. Report No. SAND2002-0835, 2002. [Online]. Available: http://citeseerx.ist.psu.edu/viewdoc/download?doi=10.1.1.122. $7929\{\&\}$ rep=rep1 $\{\&\}$ type $=$ pdf

[75] R. R. Yager, "On the dempster-shafer framework and new combination rules," Information Sciences, vol. 41, no. 2, pp. 93-137, 1987.

[76] L. Podofillini and V. N. Dang, "A Bayesian approach to treat expert-elicited probabilities in human reliability analysis model construction," Reliability Engineering and System Safety, vol. 117, pp. 52-64, 2013.

[77] A.-L. Jousselme, D. Grenier, and É. Bossé, "A new distance between two bodies of evidence," Information Fusion, vol. 2, no. 2, pp. 91-101, jun 2001. [Online]. Available: http://linkinghub.elsevier.com/retrieve/pii/S1566253501000264

[78] M. Loudahi, J. Klein, J. M. Vannobel, and O. Colot, "New distances between bodies of evidence based on Dempsterian specialization matrices and their consistency with the conjunctive combination rule," International Journal of Approximate Reasoning, vol. 55, no. 5, pp. 1093-1112, 2014. [Online]. Available: http://dx.doi.org/10.1016/j.ijar.2014.02.007

[79] F. Cuzzolin, "A geometric approach to the theory of evidence," Systems, Man, and Cybernetics, Part C: Applications and Reviews, IEEE Transactions on, vol. 38, no. 4, pp. 522-534, 2008.

[80] F. Sebbak, F. Benhammadi, M. Mataoui, S. Bouznad, and Y. Amirat, "An alternative combination rule for evidential reasoning," ... (FUSION), 2014 17th ..., 2014. [Online]. Available: http://ieeexplore.ieee.org/xpls/abs\{_\}all.jsp?arnumber=6916059

[81] L. Pickup, B. Ryan, S. Atkinson, N. Dadashi, D. Golightly, and J. Wilson, "Support Study for Human Factors Integration - Human Functions in European Railways: Final Report for the European Railway Agency," European Rail Agency, Tech. Rep. Final Report for the European Railway Agency ERA/2012/SAF/NP/02, 2013. [Online]. Available: http://www.era.europa.eu/Document-Register/Pages/Study-Human-Factors-Integration.aspx 
[82] S. Rangra, M. Sallak, W. Schön, and F. Vanderhaegen, "Human Reliability Assessment under Uncertainty - Towards a Formal Method," Procedia Manufacturing, vol. 3, no. AHFE 2015, pp. 3230-3237, 2015. [Online]. Available: http://linkinghub.elsevier.com/retrieve/pii/S2351978915008756

[83] M. Kyriakidis, "Developing a human performance railway operational index to enhance safety of railway operations," Imperial College London, no. October 2013, 2013. [Online]. Available: http://hdl.handle.net/10044/1/21760

[84] P. H. Giang and S. Shenoy, "The Belief Function Machine (BFM) An Environment for Reasoning with Belief Functions in MatLab," Univ. of Kansas, Tech. Rep., 2003. [Online]. Available: http://pshenoy.faculty.ku.edu/Papers/BFM072503.zip

[85] C. de investigación de accidentes ferroviarios, "Informe final sobre el accidente grave ferroviario $\mathrm{n}^{\mathrm{O}} 0054 / 2013$ occurido el día 24.07.2013 en las proximidades de la estación de Santiago de Compostela (A Coruña)," COMISIÓN DE INVESTIGACIÓN DE ACCIDENTES FERROVIARIOS, Tech. Rep., 2014. [Online]. Available: http://www.fomento.gob.es/NR/ rdonlyres/0ADE7F17-84BB-4CBD-9451-C750EDE06170/125127/IF240713200514CIAF.pdf

[86] D. E. Embrey, "SHERPA: A systematic human error reduction and prediction approach," in Paper Presented at the International Topical Meeting on Advances in Human Factors in Nuclear Power Systems, Knoxville, Tennessee., 1986.

Subeer Rangra received received his bachelors degree in engineering from BKBIET Pilani, India, in 2012 followed by masters in computer science specializing in safety critical software systems from ESIEE, Paris, France in 2014 . He is currently a PhD candidate in Computer Engineering Department at Heudiasyc laboratory of Université de Technologie de Compiègne, Compiègne, France, working towards the risk analysis of human factors in transportation system-of-systems (rail and automotive). He has previously worked with formal methods application in railway signaling. His research interests include safety critical systems, human factors, and railway signaling.

Mohamed Sallak received a Ph.D. degree from Polytechnic Institute of Lorraine in 2007. He has been an Associate Professor with the Computer Engineering Department at the Université de Technologie de Compiègne, Compiègne, France since 2009. He received the 'Accreditation to supervise research (HDR)' in 2015. His current research interests concern the reliability assessment of complex systems under uncertainties using several uncertainty theories (belief function theory, imprecise probability theory, and fuzzy set theory), reliability allocation methods, components importance measures, and the dependability assessment of railway systems.

Walter Schön is a graduate of the École normale supérieure (promotion 1979) Associate in Physics (1982) and Doctor in Physics (1988). From 1989 to 1998, he held various positions as Head of System Dependability Departments in the Rail Industry (Alstom and Siemens Mobility). Since 1998, he is a Professor at Université de Technologie de Compiègne, Compiègne, France, and the Head of Embedded Systems field, in Automatic Embedded Systems Robotics team of the Laboratory Heudiasyc UMR CNRS 7253, Compiègne. He is also recognized by the French Department of Transportation for his expertise in railway systems safety as part of the French notified body for railway certification Certifer. His current research interests include dependability of critical embedded systems taking into account the human factor and the uncertainties, especially for railway applications.

Frédéric Vanderhaegen received his PhD in 1993 and his Professorship Habilitation (HDR) in 2003 on "Industrial and Human Automation" at UVHC. From 1995 to 2005, he was a CNRS researcher after a post-doctorate project at the JRC of Ispra in 1994. Since 2005, his is full professor at UVHC. Since 2004, he is the head on the Human-Machine Systems (HMS) research team of the LAMIH. He is chairing several projects, conferences (e.g. IFAC/IFIP/IFORS/IEA symposia on HMS), and groups of research (e.g. CNRS GDR I HAMASYTI; IFAC TC HMS). He is the Editor-in-chief with O. Carsten of Cognition Technology \& Work. 